\title{
Asymptotics of small exterior Navier-Stokes flows with non-decaying boundary data
}

\author{
Kyungkuen Kang*, Hideyuki Miura† $\quad$ Tai-Peng Tsai ${ }^{\ddagger}$
}

2011-05-02

\begin{abstract}
We prove the unique existence of solutions of the 3D incompressible Navier-Stokes equations in an exterior domain with small non-decaying boundary data, for $t \in \mathbb{R}$ or $t \in(0, \infty)$. In the latter case it is coupled with small initial data in weak $L^{3}$. As a corollary, the unique existence of time-periodic solutions is shown for the small periodic boundary data. We next show that the spatial asymptotics of the periodic solution is given by the same Landau solution at all times. Lastly we show that if the boundary datum is time-periodic and the initial datum is asymptotically discretely self-similar, then the solution is asymptotically the sum of a timeperiodic vector field and a forward discretely self-similar vector field as time goes to infinity. It in particular shows the stability of periodic solutions in a local sense.

Keywords: Navier-Stokes equations, exterior domain, spatial asymptotics, time asymptotics, time-periodic, Landau solution, discretely self-similar, stability.

Mathematics Subject Classification (2010): 35Q30, 35B10, 35B40
\end{abstract}

\section{Introduction}

Let $\Omega \subset \mathbb{R}^{3}$ be an exterior domain with smooth boundary $\partial \Omega$ and unit outernormal $N$, and $I=\mathbb{R}$ or $I=(0, \infty)$ be the time interval. In $I \times \Omega$ we consider the nonstationary Navier-Stokes equations

$$
\begin{gathered}
\partial_{t} u-\Delta u+(u \cdot \nabla) u+\nabla p=f, \quad \operatorname{div} u=0, \\
\left.u\right|_{\partial \Omega}=u_{*}, \quad \lim _{|x| \rightarrow \infty} u=0,
\end{gathered}
$$

where $u(t, x): \bar{I} \times \Omega \rightarrow \mathbb{R}^{3}$ is the unknown velocity field, $p(t, x): \bar{I} \times \Omega \rightarrow \mathbb{R}$ the unknown pressure, $u_{*}$ the given boundary data and $f=f_{0}+\nabla \cdot F$ the given force with $F=\left(F_{i j}\right)$ being a 2-tensor and $(\nabla \cdot F)_{j}=\partial_{i} F_{i j}$. We use summation convention for repeated index and $u \cdot \nabla=u_{j} \partial_{j}$. In the case $I=(0, \infty)$, we add the initial condition

$$
\left.u\right|_{t=0}=u_{0}, \quad \operatorname{div} u_{0}=0 .
$$

By an exterior domain we mean a connected open set with bounded complement. Without loss of generality, we may assume the complement of $\bar{\Omega}$ contains the origin and is a subset of $B_{R_{1}}:=\{x \in$ $\left.\mathbb{R}^{3}:|x|<R_{1}\right\}$ for some $R_{1}>0$.

In this paper, we first consider the solvability of the problem (1.1)-(1.3) with nondecaying boundary data in time. Moreover, we also study the asymptotic properties of solutions with time-periodic boundary data.

To explain the background, we start with the review of the stationary problem. In 1965 Finn 9 showed the existence of a small stationary solution satisfying $|u(x)| \leq C|x|^{-1}$ in $\Omega$ for small data. Nazarov and Pileckas [27] proved that the solution is asymptotically self-similar at spatial infinity, i.e., the solution converges to a (-1)-homogeneous vector field faster than $C|x|^{-1}$. Recently Korolev

\footnotetext{
*Department of Mathematics, Yonsei University, Seoul 120-749, South Korea. Email: kkang@yonsei.ac.kr

$\dagger$ Department of Mathematics, Osaka University, Osaka, Japan. Email: miura@math.sci.osaka-u.ac.jp

$\ddagger$ Department of Mathematics, University of British Columbia, Vancouver, BC V6T 1Z2, Canada. Email: ttsai@math.ubc.ca.
} 
and Sverak [17] showed the asymptotic profile is given by a Landau solution. In particular the decay rate $|x|^{-1}$ is optimal in general.

Landau solutions are a family of vector fields $U^{b}$ and functions $P^{b}$ in $\mathbb{R}^{3}$, with parameter $b \in \mathbb{R}^{3}$, that solve

$$
-\Delta u+(u \cdot \nabla) u+\nabla p=b \delta_{0}, \quad \operatorname{div} u=0,
$$

where $\delta_{0}$ is the delta function at the origin. They are axisymmetric: in spherical coordinates $\rho, \theta, \varphi$ with $b$ in the direction of the north pole,

$$
U^{b}=\frac{2}{\rho}\left(\frac{A^{2}-1}{(A-\cos \varphi)^{2}}-1\right) e_{\rho}+0 e_{\theta}-\frac{2 \sin \varphi}{\rho(A-\cos \varphi)} e_{\varphi},
$$

where $A=A(|b|) \in(1, \infty]$ is determined by $|b|=16 \pi\left(A+\frac{1}{2} A^{2} \log \frac{A-1}{A+1}+\frac{4 A}{3\left(A^{2}-1\right)}\right)$ and strictly decreasing in $|b| \geq 0$. It is the unique solution of (1.4) in the class of $(-1)$-homogeneous vector fields in $\mathbb{R}^{3}$. See Landau [22, 23], Tian and Xin [36], and Sverak [34].

On the other hand, there are also a lot of works on the time-periodic solutions in exterior domains. After the earlier works by Salvi [30] and Maremonti-Padula [24, Yamazaki 37] showed the unique existence of time-periodic solutions in the Lorentz space $L^{3, \infty}$ for zero boundary data and small forces $F \in L^{3 / 2, \infty}$ with $f_{0}=0$. Galdi and Sohr 12 further showed the existence of time-periodic solutions satisfying the pointwise estimate $|u(t, x)| \leq C|x|^{-1}$ where $C$ is independent of time.

When we are interested in time-periodic solutions or, more generally, the exterior problem for nondecaying boundary data and forces, the function spaces should allow nondecaying functions in time. On the other hand, in view of the optimal decay rate $|x|^{-1}$ for the stationary solutions, it is natural to choose the spaces $L^{3, \infty}$ (weak- $L^{3}$ space) or $X_{1}$ in spatial variables, where $X_{k}$ (for $k>0$ ) is the space of functions defined by the norm

$$
\|u\|_{X_{k}}:=\sup _{x \in \Omega}(1+|x|)^{k}|u(x)| .
$$

Let $B C_{w}(J ; X)$ be the class of bounded and weak-star continuous $X$-valued functions defined on a time interval $J$. We omit the subscript $w$ if it is strongly continuous.

Our first result concerns the unique existence of very weak solutions, to be defined in $\$ 2.2$, with small nondecaying boundary data in the time interval $\mathbb{R}$ or $(0, \infty)$. As a consequence, we obtain an existence theorem of time-periodic solutions.

Theorem 1.1 (Existence) Let $\Omega$ be a smooth exterior domain in $\mathbb{R}^{3}$ with $\partial \Omega \subset\left\{x:|x|<R_{1}\right\}$. Let $I$ be $\mathbb{R}$ or $(0, \infty)$. Let $\delta$ be a positive constant. There are constants $\varepsilon_{0}>\tilde{\varepsilon}_{0}>0$ and $C>0$ such that the following holds: Let $u_{0}, u_{*}, f_{0}$ and $F$ be given data with the convention $u_{0}=0$ if $I=\mathbb{R}$.

(i) Assume that

$$
\varepsilon:=\left\|u_{*}\right\|_{W^{1, \infty}\left(I ; C_{x}^{2}(\partial \Omega)\right)}+\left\|f_{0}\right\|_{L^{\infty}\left(I ; X_{3+\delta}\right)}+\|F\|_{L^{\infty}\left(I ; L^{3 / 2, \infty}(\Omega)\right)}+\left\|u_{0}\right\|_{L^{3, \infty}(\Omega)} \leq \varepsilon_{0} .
$$

Then there is a unique very weak solution $u \in B C_{w}\left(\bar{I} ; L^{3, \infty}(\Omega)\right)$ of (1.1)-(1.3) satisfying

$$
\|u\|_{L^{\infty}\left(I ; L^{3, \infty}(\Omega)\right)} \leq C \varepsilon .
$$

(ii) Let $I=\mathbb{R}$. Assume that all $u_{*}, f_{0}$ and $F$ are periodic in time with period $T>0$, then the solution in (i) is also periodic in time with same period.

(iii) Let $I=\mathbb{R}$. Assume that

$$
\tilde{\varepsilon}:=\left\|u_{*}\right\|_{W^{2, \infty}\left(\mathbb{R} ; C_{x}^{2}(\partial \Omega)\right)}+\left\|f_{0}\right\|_{W^{1, \infty}\left(\mathbb{R} ; X_{3+\delta}\right)}+\|F\|_{W^{1, \infty}\left(\mathbb{R} ; X_{2}\right)} \leq \tilde{\varepsilon}_{0},
$$

then the solution in (i) satisfies

$$
|u(t, x)| \leq C \tilde{\varepsilon}|x|^{-1}, \quad\left(|x|>R_{1}, t \in \mathbb{R}\right) .
$$

\section{Comments for Theorem 1.1:}

1. The initial condition (1.3) is understood in the weak-star sense. Thus we do not need a compatibility condition between $u_{*}$ and $u_{0}$. When $u_{*}=0$, we usually also require $u$ belongs $L_{\sigma}^{3, \infty}(\Omega)$ which is the subspace of $L^{3, \infty}\left(\Omega ; \mathbb{R}^{3}\right)$ with $\operatorname{div} u=0$ and $\left.u \cdot N\right|_{\partial \Omega}=0, N$ being the outer-normal of $\Omega$, see 2.1 This is not suitable for the inhomogeneous boundary value problem. 
2. In the case $I=\mathbb{R}$ and $u_{*}$ is time-independent or time-periodic, we recover the results of Finn [9, Galdi and Sohr [12] on the existence of solutions behaving like $|x|^{-1}$ as $|x| \rightarrow \infty$.

3. As noted above, there are a lot of literature on the existence of periodic exterior flows for zero boundary data [30, 24, 37, 12. Our result allows nonzero boundary data. There are also results on existence of solutions for nonzero boundary data e.g. Amann 2], Farwig, Kozono and Sohr [8]. However, most of them require time decay of the boundary data, which is not suitable for the periodic solutions. Since the maximal regularity estimates in [2, 8] are not available in our solution spaces, we use the duality argument by Yamazaki [37 to construct the solution. In fact, we first decompose $u=E+v$ where $E$ is an extension of the boundary data $u_{*}$, and then we construct the unique mild solution $v$ of the difference equation (3.8) following the method by 37. Unlike [37, we do not require time continuity of the force (in part (i)) and our solution is only weak-star continuous in $t$. We then show the equivalence of this solution and a very weak solution in $\$ 3.2$ Note the datum $u_{*}$ is in the $C^{2}$ class, not usual $C^{2, \alpha}, 0<\alpha<1$. The existence of the extension $E$ is shown in Lemma 2.4.

Our second result concerns the spatial asymptotics of time-periodic solutions. To describe it, we recall the momentum flux density tensor for a solution $(u, p)$ of (1.1),

$$
T_{i j}(u, p, F)=p \delta_{i j}+u_{i} u_{j}-\partial_{i} u_{j}-\partial_{j} u_{i}-F_{i j}
$$

Equation (1.1) can be written as

$$
\partial_{t} u_{j}+\partial_{i} T_{i j}=f_{0 j}, \quad \operatorname{div} u=0 .
$$

We show that the asymptotic profile of a time-periodic solution is given by a Landau solution determined by the tensor $T_{i j}$ and chosen independent of time.

Theorem 1.2 (Spatial asymptotics of time-periodic solutions) For any $T>0, R>0$ and $\alpha \in(1,2)$, there are constants $\varepsilon_{1}>0$ and $C>0$ such that the following holds. Suppose $(u, p)$ is a time-periodic solution of (1.1) with period $T$ for $R<|x|<\infty$, and satisfies

$$
\begin{gathered}
\varepsilon:=\sup _{t \in \mathbb{R}}\left\{\sup _{|x|>R}\left\{|x|^{2+\alpha}\left|f_{0}(t, x)\right|+|x|^{1+\alpha}|F(t, x)|\right\}+\|u(t, \cdot)\|_{L^{3, \infty}(|x|>R)}+\right. \\
\left.+\sup _{R<|x|<R+1}\{|u(t, x)|+|\nabla u(t, x)|+|p(t, x)|\}\right\} \leq \varepsilon_{1} .
\end{gathered}
$$

Then $|u(t, x)| \leq C \varepsilon|x|^{-1}$ for $|x|>R$ and all $t \in \mathbb{R}$. Moreover, let $T_{i j}$ be defined by (1.11), let

$$
b_{j}=\lim _{\rho \rightarrow \infty} \frac{1}{T} \int_{0}^{T} \int_{|x|=\rho} T_{i j} n_{i} d S_{x} d t, \quad\left(n_{i}=\frac{x_{i}}{|x|}, i=1,2,3\right),
$$

and let $U^{b}$ be the Landau solution corresponding to $b$, whose choice is independent of $t$. Then

$$
\left|u(t, x)-U^{b}(x)\right| \leq C \varepsilon|x|^{-\alpha}, \quad(|x|>R ; t \in \mathbb{R}) .
$$

Comments for Theorem 1.2:

1. In Theorem 1.2 we do not specify a boundary condition for $u$. All we need is a solution for $|x|>R$. Thus our result is applicable to the periodic solutions constructed in Theorem 1.1 (ii), (iii).

2. If $u$ is independent of time, Theorem 1.2 recovers the result of Korolev-Sverak [17] for small exterior stationary Navier-Stokes flows. We observe that the decay rate $|u(t, x)| \leq C|x|^{-1}$ for small periodic solutions is optimal as well as the stationary case.

3. In order to see that the limit in (1.14) exists, denote the integral as $I_{j}(\rho)$ and then we have from (1.12) that

$$
I_{j}\left(\rho_{2}\right)-I_{j}\left(\rho_{1}\right)=\frac{1}{T} \int_{0}^{T} \int_{\rho_{1}<|x|<\rho_{2}} f_{0 j} \quad\left(R<\rho_{1}<\rho_{2}\right) .
$$

We would like to emphasize that the choice of $b$ in (1.15) is independent of $t$. There is some sort of cancellation effect behind it. 
Finally we consider the large time asymptotics of solutions of the problem (1.1)-1.3) when the boundary data and the force are time-periodic. Borchers and Miyakawa 3 showed the stability of stationary exterior flows under small initial perturbation in $L^{3, \infty}$ vanishing at boundary. For earlier stability results, see e.g. [14, 19]. Our third theorem extends these stability results to small time-periodic solutions.

Theorem 1.3 (Time asymptotics) For any $T>0, \delta>0, \eta>0$ and $3 \leq q_{1}<\frac{3}{\delta+\eta}$, there is $\varepsilon_{2}>0$ such that the following holds. Let $u_{*}$ and $f$ be time-periodic data satisfying (1.9). Assume the initial data $u_{0}$ is asymptotically self-similar in the sense that there is a (-1)-homogeneous vector field $\tilde{u}_{0} \in X_{1}$ such that $\operatorname{div} \tilde{u}_{0}=0$ and

$$
\left\|\tilde{u}_{0}\right\|_{X_{1}} \leq \varepsilon_{2}, \quad \nabla \tilde{u}_{0} \in X_{2} \quad \text { and } \quad \tilde{\varepsilon}:=\left\|u_{0}-\tilde{u}_{0}\right\|_{L^{3, \infty} \cap L^{\frac{3}{1+\delta}}, \infty} \leq \varepsilon_{2} .
$$

Then the solution $u$ in Theorem 1.1 (i) for $t \geq 0$ can be decomposed as

$$
u=Q+w+r
$$

Here $Q$ is the periodic solution for the data $u_{*}$ and $f$ in Theorem 1.1 (iii). The term $w$ is the forward self-similar solution of the perturbed Navier-Stokes system in $(0, \infty) \times \mathbb{R}^{3}$,

$$
\partial_{t} w-\Delta w+\nabla\left(w \otimes w+U^{b} \otimes w+w \otimes U^{b}\right)+\nabla p_{0}=0, \quad \operatorname{div} w=0,
$$

with initial data $w_{0}=\tilde{u}_{0}-U^{b}$, where $U^{b}(x)$ is the Landau solution corresponding to $Q$ given in Theorem 1.2 with $\alpha=1+\delta$, and $w$ satisfies

$$
|w(t, x)| \leq C \varepsilon_{2}(|x|+\sqrt{t})^{-1+\eta}|x|^{-\eta}, \quad\left(t \in(0, \infty) ; x \in \mathbb{R}^{3}\right) .
$$

The term $r$ satisfies the following decay estimate:

$$
\|r(t)\|_{L^{q, \infty}(\Omega)} \leq C \tilde{\varepsilon} t^{-\frac{3}{2}\left(\frac{1}{3}-\frac{1}{q}\right)-\frac{\delta}{2}} \quad \forall t>0, \quad \forall q \in\left[3, q_{1}\right] .
$$

Comments for Theorem 1.3:

1. The last assumption in (1.17) means that the profile of $u_{0}$ at spatial infinity is given by $\tilde{u}_{0}$. Since $\|Q(t, x)\|_{L^{q, \infty}} \leq C,\|w(t, x)\|_{L^{q, \infty}} \leq C t^{-\frac{3}{2}\left(\frac{1}{3}-\frac{1}{q}\right)}$ for $3 \leq q \leq q_{1}$ and $r$ decays faster than the other terms as $t \rightarrow \infty$, our theorem shows that $u$ is asymptotically equal to the sum of $Q$ and $w$. In particular, this implies the stability of the periodic solution.

2. In the case $\Omega=\mathbb{R}^{3}$ with zero force and no boundary data, Planchon 29 showed that if the initial data is asymptotically self-similar, the solution is also asymptotically self-similar. Here the asymptotic profile of the solution at large time is given by the self-similar solution of the non-perturbed equations (1.1) with zero force. See also 77. In our case, the asymptotic profile $w$ satisfies an equation modified by the Landau solution $U^{b}$, but it is still self-similar. Unique existence of the self-similar solutions for (1.19) is considered in Proposition 5.1

3. If $\tilde{u}_{0}$ is not self-similar, we still have similar decomposition like (1.19). In particular, if $u_{0}$ is asymptotically discretely self-similar, $w$ becomes forward discretely self-similar. The notion of the discretely self-similar solution is discussed in the introduction of section 5 . In section 6 . we will show a more general decomposition. See Theorem 6.1.

Our previous three theorems can be similarly posed in the entire $\mathbb{R}^{3}$ with a singular force supported at the origin

$$
\partial_{t} u-\Delta u+(u \cdot \nabla) u+\nabla p=b(t) \delta_{0}, \quad \operatorname{div} u=0, \quad\left(x \in \mathbb{R}^{3}\right),
$$

which is studied by Cannone and Karch [4. The problem will be pursued elsewhere.

This paper is organized as follows. In section 2, we recall some preliminary results related to the exterior problem. In section 3 we prove Theorem 1.1. Section 4 is devoted to the proof of Theorem 1.2. In section [5 we consider the perturbed Navier-Stokes system (1.19) which plays an important role in the next section. Finally we consider the time asymptotics of the exterior flows and prove Theorem 1.3 in section 6 .

Notation. $a \lesssim b$ means $a \leq C b$ for some constant $C . a \sim b$ means $a \lesssim b \lesssim a .\langle a\rangle=\sqrt{|a|^{2}+1}$. For $1 \leq p \leq \infty$, its conjugate exponent $p^{\prime}$ is defined by $1 / p+1 / p^{\prime}=1$. We denote $\mathbb{R}_{+}=(0, \infty)$. 


\section{Preliminaries}

In this preparation section we recall Helmholtz decomposition and Stokes semigroup in $\$ 2.1$, define very weak solutions in $\$ 2.2$, prove extension lemmas in $\$ 2.3$, show that $f_{0}$ can be absorbed into $\nabla F$ in $\$ 2.4$ and prove decay estimates for Stokes system in $\$ 2.5$.

Recall $\Omega$ denotes an exterior domain in $\mathbb{R}^{3}$ with smooth boundary $\partial \Omega$ and unit outer-normal $N$. We also assume $0 \notin \bar{\Omega}$ and $\partial \Omega \subset B_{R_{1}}$.

\subsection{Helmholtz decomposition and Stokes operator}

The Helmholtz decomposition

$$
L^{q}\left(\Omega ; \mathbb{R}^{n}\right)=L_{\sigma}^{q}(\Omega) \oplus G^{q}(\Omega), \quad(1<q<\infty),
$$

for a $C^{1}$-exterior domain $\Omega \subset \mathbb{R}^{n}, n \geq 2$, is well understood. It is first proved for $n=3$ by Miyakawa [26] and for all $n \geq 2$ by Simader-Sohr [31. Let $P=P_{q}$ be the associated Helmholtz projector from $L^{q}$ onto $L_{\sigma}^{q}$. Then $P$ can be extended by interpolation to a bounded projector on each Lorentz space $L^{q, r}(\Omega), 1<q<\infty, 1 \leq r \leq \infty$, with the Helmholtz decomposition

$$
L^{q, r}\left(\Omega ; \mathbb{R}^{n}\right)=L_{\sigma}^{q, r}(\Omega) \oplus G^{q, r}(\Omega)
$$

where

$$
\begin{aligned}
L_{\sigma}^{q, r}(\Omega) & =\left\{u \in L^{q, r}\left(\Omega ; \mathbb{R}^{n}\right): \operatorname{div} u=0,\left.u \cdot N\right|_{\partial \Omega}=0\right\}, \\
G^{q, r}(\Omega) & =\left\{\nabla p \in L^{q, r}\left(\Omega ; \mathbb{R}^{n}\right): p \in L_{l o c}^{q, r}(\bar{\Omega})\right\} .
\end{aligned}
$$

Furthermore, if $1 \leq r<\infty$, then $\left(L_{\sigma}^{q, r}\right)^{*}=L_{\sigma}^{\left(q^{\prime}, r^{\prime}\right)}$ and $\left(G^{q, r}\right)^{*}=G^{\left(q^{\prime}, r^{\prime}\right)}$. See [3, Th. 5.2].

The Stokes operator $A_{q}$ on $L_{\sigma}^{q}(\Omega)$ with the dense domain

$$
\mathcal{D}\left(A_{q}\right)=L_{\sigma}^{q}(\Omega) \cap W_{0}^{1, q}(\Omega) \cap W^{2, q}(\Omega)
$$

is defined by $A_{q} u=-P_{q} \Delta u$ for $u \in \mathcal{D}\left(A_{q}\right)$. It extends to a closed linear operator on $L_{\sigma}^{q, r}(\Omega)$ with domain

$$
\mathcal{D}\left(A_{q, r}\right)=\left\{u \in L_{\sigma}^{q, r}(\Omega): \nabla^{j} u \in L^{q, r}(\Omega), j=1,2,\left.u\right|_{\partial \Omega}=0\right\} .
$$

One also has

$$
\mathcal{D}\left(A_{q, r}^{1 / 2}\right)=L_{\sigma}^{q, r}(\Omega) \cap W_{0}^{1,(q, r)}(\Omega) .
$$

The semigroup $\left\{e^{-t A}\right\}_{t \geq 0}$ also extends to $L_{\sigma}^{q, r}(\Omega)$.

We now recall some estimates in Lorentz spaces.

Lemma 2.1 Let $\Omega \subset \mathbb{R}^{3}$ be an exterior domain with smooth boundary. One has

$$
\begin{aligned}
\left\|A^{1 / 2} u\right\|_{L^{p, r}} & \lesssim\|\nabla u\|_{L^{p, r}}, \quad(1<p<\infty, 1 \leq r \leq \infty), \\
\|\nabla u\|_{L^{p, \infty}} & \lesssim\left\|A^{1 / 2} u\right\|_{L^{p, \infty}}, \quad(1<p<3), \\
\|\nabla u\|_{L^{p, 1}} & \lesssim\left\|A^{1 / 2} u\right\|_{L^{p, 1}}, \quad(1<p \leq 3) .
\end{aligned}
$$

The above is [37, Theorem 2.1].

For $1 \leq p<3$, we define $p^{*}$ by $1 / p^{*}=1 / p-1 / 3$.

Lemma 2.2 Let $\Omega \subset \mathbb{R}^{3}$ be an exterior domain with smooth boundary.

(i) For $1<p \leq q<\infty, \sigma=\frac{3}{2}\left(\frac{1}{p}-\frac{1}{q}\right)$ and $1 \leq r \leq \infty$, for any $\phi \in L_{\sigma}^{p, r}(\Omega)$

$$
\begin{gathered}
\left\|e^{-t A} \phi\right\|_{L^{q, r}} \lesssim t^{-\sigma}\|\phi\|_{L^{p, r},} \quad t>0, \\
\left\|A^{1 / 2} e^{-t A} \phi\right\|_{L^{q, r}} \lesssim t^{-\sigma-\frac{1}{2}}\|\phi\|_{L^{p, r}}, \quad t>0 .
\end{gathered}
$$

(ii) For $1<p<3$, for some $c=c(\Omega, p)>0$, for any $\phi \in L_{\sigma}^{p, 1}(\Omega)$,

$$
\int_{0}^{\infty}\left\|A^{1 / 2} e^{-t A} \phi\right\|_{L^{p^{*}, 1}} d t \leq c\|\phi\|_{L^{p, 1}}
$$


(iii) For $1<p<3$ and $0<\sigma<1$, for some $c=c(\Omega, p, \sigma)>0$, for any $\phi \in L_{\sigma}^{p, 1}(\Omega)$,

$$
\int_{0}^{t} s^{-\sigma}\left\|A^{1 / 2} e^{-(t-s) A} \phi\right\|_{L^{p^{*}, 1}} d s \leq c t^{-\sigma}\|\phi\|_{L^{p, 1}}, \quad t>0 .
$$

If $1<p \leq \frac{3}{2}$, we may replace $A^{1 / 2}$ in (2.12) and (2.13) by $\nabla$ due to (2.9).

Proof. The decay estimates (2.10) amd (2.11) are [37, (2.4), (2.5)]. (Although [37] only states the case $r=1$, it works for other $r$.) The integral estimate (2.12) follows from [37, (2.11)].

To show (2.13), divide the integral to $s \leq t / 2$ and $s>t / 2$. Applying (2.11) for $s \leq t / 2$ and (2.12) for $s>t / 2$, we get (2.13).

As a corollary, we have the following lemma. Here $B C_{w}$ denotes the class of bounded and weak-star continuous functions.

Lemma 2.3 Let $\Omega \subset \mathbb{R}^{3}$ be an exterior domain with smooth boundary. Let $I=\mathbb{R}$ or $I=\mathbb{R}_{+}$. For $f(t) \in L^{\infty}\left(I ; L^{q, \infty}(\Omega)\right), 3 / 2 \leq q<3$, define

$$
\left(\mathcal{G}_{I} f\right)(t)=\int_{\inf I}^{t} e^{-(t-s) A} P \nabla f(s) d s, \quad(t \in I)
$$

in the sense that, for $p=\left(q^{*}\right)^{\prime}$, that is, $1 / p=4 / 3-1 / q$,

$$
\left(\left(\mathcal{G}_{I} f\right)(t), \phi\right)=\int_{\inf I}^{t}\left(f(s), \nabla e^{-(t-s) A} \phi\right) d s, \quad \forall \phi \in L_{\sigma}^{p, 1}, \forall t \in I .
$$

Then $\mathcal{G}_{I} f \in B C_{w}\left(\bar{I} ; L_{\sigma}^{q^{*}}, \infty(\Omega)\right)$, and for some $c=c(\Omega, q)>0$

$$
\left\|\mathcal{G}_{I} f\right\|_{L^{\infty}\left(I ; L_{\sigma}^{q^{*}, \infty}\right)} \leq c\|f\|_{L^{\infty}\left(I ; L^{q, \infty}\right)}
$$

Note the integral $\mathcal{G}_{I} f$ is in weak sense and may not converge absolutely.

Proof. For any $\phi \in L_{\sigma}^{p, 1}$ and $t \in I$, we have

$$
\begin{aligned}
\left|\left(\mathcal{G}_{I} f(t), \phi\right)\right| & =\left|\int_{0}^{t-\inf I}\left(f(t-\tau), \nabla e^{-\tau A} \phi\right) d \tau\right| \\
& \leq \int_{0}^{\infty}\|f(t-\tau)\|_{L^{q, \infty}}\left\|\nabla e^{-\tau A} \phi\right\|_{L^{q^{\prime}, 1}} d \tau .
\end{aligned}
$$

Note that $3 / 2 \leq q<3$ is equivalent to $1<p \leq 3 / 2$ and that $q^{\prime}=p^{*}$. Then Lemma 2.2 (ii) implies that

$$
\left|\left(\mathcal{G}_{I} f(t), \phi\right)\right| \leq c\|f\|_{L^{\infty}\left(I ; L^{q, \infty}\right)}\|\phi\|_{L^{p, 1}},
$$

which shows $\mathcal{G}_{I} f \in L^{\infty}\left(I ; L_{\sigma}^{q^{*}, \infty}(\Omega)\right)$ and (2.16). To show weak continuity, a computation similar to the above shows $\forall t<t^{\prime} \in I$ and $\varepsilon:=t^{\prime}-t$

$$
\begin{aligned}
& \left|\left(\mathcal{G}_{I} f\left(t^{\prime}\right)-\mathcal{G}_{I} f(t), \phi\right)\right| \\
& \leq \int_{0}^{\varepsilon}\left|\left(f\left(t^{\prime}-\tau\right), \nabla e^{-\tau A} \phi\right)\right| d \tau+\int_{0}^{\infty}\left|\left(f(t-\tau), \nabla e^{-\tau A}\left(e^{-\varepsilon A}-1\right) \phi\right)\right| d \tau \\
& \leq\|f\|_{L^{\infty} L^{q, \infty}}\left(\int_{0}^{\varepsilon}\left\|\nabla e^{-\tau A} \phi\right\|_{L^{q^{\prime}, 1}} d \tau+\int_{0}^{\infty}\left\|\nabla e^{-\tau A}\left(e^{-\varepsilon A}-1\right) \phi\right\|_{L^{q^{\prime}, 1}} d \tau\right) .
\end{aligned}
$$

By Lemma 2.2 and by the strong continuity at $\varepsilon=0$ of the Stokes semigroup in $L^{p, 1}$, the right hand side converges to 0 as $\varepsilon \rightarrow 0_{+}$, and hence $\left|\left(\mathcal{G}_{I} f\left(t^{\prime}\right)-\mathcal{G}_{I} f(t), \phi\right)\right| \rightarrow 0$ with either $t$ or $t^{\prime}$ fixed.

\subsection{Very weak solutions}

In this subsection we define very weak solutions in an exterior domain $\Omega$ with unit outernormal $N$. Our definition is a variant of that in Amann [2] and Farwig, Kozono and Sohr [8]. We take the constant $\kappa$ to be 1 for the Navier-Stokes system, and $\kappa=0$ for the Stokes system. 
For finite or infinite time interval $J$, denote the space of test functions

$$
\mathcal{D}_{J}:=\left\{w \in C\left(\Omega_{J} ; \mathbb{R}^{3}\right): \begin{array}{c}
\partial_{t}^{a} \nabla_{x}^{b} w \in C\left(\Omega_{J}\right),(a \leq 1 ; b \leq 2) ; \\
\operatorname{div} w=0, \operatorname{supp} w \Subset \Omega_{J},\left.w\right|_{\partial \Omega}=0
\end{array}\right\},
$$

where $\Omega_{J}=\bar{J} \times \bar{\Omega}$ contains the boundary. Thus $\nabla w$ may be nonzero on boundary, and $w\left(t_{0}, \cdot\right)$ may be nonzero if $t_{0}=\inf J$ is finite.

Let $I=\mathbb{R}$ or $I=\mathbb{R}_{+}$. A vector field $u \in L_{\text {loc }}^{2}\left(\bar{I} \times \bar{\Omega} ; \mathbb{R}^{3}\right)$ is called a very weak solution of the Navier-Stokes system (when $\kappa=1$ ) or of the Stokes system (when $\kappa=0$ ) with initial datum $u_{0}$ (with the convention $u_{0}=0$ if $I=\mathbb{R}$ ), boundary datum $u_{*}$, force $f$ and mass source $k$ (with sufficient regularity) if

$$
\begin{aligned}
& \int_{I}\left\{-\left(u, w_{t}+\Delta w\right)_{\Omega}+\left(u_{*}, N \cdot \nabla w\right)_{\partial \Omega}-(\kappa u \otimes u, \nabla w)_{\Omega}-(k u, w)_{\Omega}\right\} d t \\
& =\left(u_{0}, w(0)\right)_{\Omega}+\int_{I}(f, w)_{\Omega} d t, \quad \forall w \in \mathcal{D}_{I},
\end{aligned}
$$

and

$$
\operatorname{div} u(t)=k(t),\left.\quad N \cdot u(t)\right|_{\partial \Omega}=N \cdot u_{*}(t) \text { for a.e. } t \in I .
$$

An elementary calculation shows that for $w \in \mathcal{D}_{J}$

$$
N \cdot \nabla w=\operatorname{curl} w \times N \quad \text { on } \partial \Omega .
$$

Thus (2.21) contains a condition only on the tangential component $N \times u_{*}$ of $u_{*}$ on $\partial \Omega$, and we have to assume the additional condition in (2.22) for the normal component $\left.N \cdot u\right|_{\partial \Omega}=N \cdot u_{*}$. Note that, when $\Omega$ is a bounded domain, one needs to assume the compatibility condition $\int_{\partial \Omega} u_{*}(t) \cdot N d S=\int_{\Omega} k(t) d x$ for a.e. $t$. When $\Omega$ is an exterior domain, however, this is unnecessary. For the rest of this paper we take $k=0$.

\subsection{Extension lemmas}

We will use the following extension lemma to extend a given boundary data $u_{*}$ with zero flux on every connected component of $\partial \Omega$ to a divergence-free vector field of compact support in $\Omega$. The special case we need is $u_{*} \in C^{2}(\partial \Omega)$ and $\Omega$ is a smooth exterior domain in $\mathbb{R}^{3}$.

Lemma 2.4 Let $l \in \mathbb{N}$ and $0 \leq \alpha \leq 1$. Assume $\Omega$ is a domain in $\mathbb{R}^{n}, n \geq 2$, with compact boundary $\partial \Omega$ of class $C^{l+1, \alpha}$ and unit outernormal $N$. Suppose $\partial \Omega$ has $M$ connected components $\Gamma_{k}, k=1, \ldots, M$. For any $\delta>0$, there is a linear map $\mathcal{E}$ which assigns for each $u_{*} \in C^{l, \alpha}\left(\partial \Omega, \mathbb{R}^{n}\right)$ with $\int_{\Gamma_{k}} u_{*} \cdot N=0$ for all $k$, a vector field $u=\mathcal{E}\left(u_{*}\right) \in C^{l, \alpha}(\bar{\Omega})$ so that

$$
\operatorname{div} u=0,\left.\quad u\right|_{\partial \Omega}=u_{*}, \quad \operatorname{supp} u \subset \bar{\Omega}_{\delta}, \quad\|u\|_{C^{l, \alpha}(\bar{\Omega})} \leq C\left\|u_{*}\right\|_{C^{l, \alpha}(\partial \Omega)} .
$$

Above $\Omega_{\delta}=\{x \in \Omega: \operatorname{dist}(x, \partial \Omega)<\delta\}$ and $C=C(\Omega, l, \alpha, \delta)$. This linear map restricted to smooth $u_{*}$ is the same for all $(l, \alpha)$ so that $\partial \Omega \in C^{l+1, \alpha}$.

The following is a more general result which we will not use.

Lemma 2.5 Let $l \in \mathbb{N}$ and $0 \leq \alpha \leq 1$. Assume $\Omega$ is a domain in $\mathbb{R}^{n}, n \geq 2$, with compact boundary $\partial \Omega$ of class $C^{l+1, \alpha}$ and unit outernormal $N$. For any open convex set $\omega \subset \mathbb{R}^{n}$ containing $\partial \Omega$, there is a linear map $\mathcal{E}$ which assigns for each $u_{*} \in C^{l, \alpha}\left(\partial \Omega, \mathbb{R}^{n}\right)$ with $\int_{\partial \Omega} u_{*} \cdot N=0$, a vector field $u=\mathcal{E}\left(u_{*}\right) \in C^{l, \alpha}(\bar{\Omega})$ so that

$$
\operatorname{div} u=0,\left.\quad u\right|_{\partial \Omega}=u_{*}, \quad \operatorname{supp} u \subset \bar{\Omega} \cap \omega, \quad\|u\|_{C^{l, \alpha}(\Omega)} \leq C\left\|u_{*}\right\|_{C^{l, \alpha}(\partial \Omega)}
$$

This linear map restricted to smooth $u_{*}$ is the same for all $(l, \alpha)$ so that $\partial \Omega \in C^{l+1, \alpha}$.

Remarks on Lemmas 2.4 and 2.5: 
1. If $u_{*} \in C^{2, \alpha}(\partial \Omega), 0<\alpha<1$, Lemmas 2.4 and 2.5 (Lemma 2.5] in the case $\Omega$ is bounded) is proved in Kapitanskii-Piletskas 15. In addition to induction in dimension, they write $u_{i}=\partial_{i}\left(w_{i j}+\delta_{i j} \phi\right)$ with $w_{i j}$ anti-symmetric and estimate Newtonian potentials in Hölder spaces. This is why they do not allow $\alpha=0$ or $\alpha=1$, which are allowed in Lemmas 2.4 and 2.5

2. In case $\Omega$ is an exterior domain, the extension constructed in [15] does not have compact support unless one further assumes $\int_{\Gamma_{k}} u_{*} \cdot N=0$ for all $k$. See also Kozono-Yanagisawa [21] for extensions in more general domains.

3. A related problem is the construction of a vector field $v$ for a given $f: \Omega \rightarrow \mathbb{R}$ satisfying $\int_{\Omega} f=0$ so that

$$
\operatorname{div} v=f, \quad(x \in \Omega),\left.\quad v\right|_{\partial \Omega}=0, \quad\|\nabla v\| \leq C\|f\|
$$

for suitable norms. The extension problem is reduced to (2.26) as follows: first extend $u_{*}$ to $U \in C^{k, \alpha}(\Omega)$ which may not be divergence-free, then solve 2.26 with $f=\operatorname{div} U$, and finally define $u=U-v$. The problem (2.26) is solved first for $f \in C_{c}^{\infty}(\Omega)$ and then for general $f$ by approximation. For $1<q<\infty$, this approach is good for $u_{*} \in W^{1-1 / q, q}(\partial \Omega)$, but not suitable for $u_{*} \in W^{k-1 / q, q}(\partial \Omega), k \geq 2$, since $C_{c}^{\infty}(\Omega)$ is not dense in $W^{k, q} \cap W_{0}^{1, q}$. Similarly it is not suitable for $u_{*} \in C^{2}(\partial \Omega)$.

4. Remark VIII.4.1 on [11, p25] says that there is no linear map which assigns a $v$ satisfying (2.26) for a given $f$ so that $\|v\|_{L^{q}} \leq c\|f\|_{W^{-1, q}}$, which we fully agree. However, we do not understand why it continues to assert that, "by the same token", there is no linear map which assigns a $v$ for $f \in C(\bar{\Omega})$ so that $\|v\|_{C^{1}} \leq c\|f\|_{C}$.

5. Theorem 4 of $[15$ asserts the existence a solution $v$ of $(2.26)$ satisfying (for $0<\alpha<1$ )

$$
\|v\|_{C^{k+1, \alpha}(\Omega)} \leq C\|f\|_{C^{k, \alpha}(\Omega)} .
$$

However, its proof assumes $\operatorname{dist}(\operatorname{supp} f, \partial \Omega)>0$ and the constant $C$ depends on this distance. See [15, §6].

Proof of Lemma 2.4. We will prove the case $n=3$ for notational simplicity. The general case $(n \geq 2)$ is proved in the same way. Denote $x^{\prime}=\left(x_{1}, x_{2}\right)$ and the open square $K=\left\{x^{\prime} \in \mathbb{R}^{2}:-1<\right.$ $\left.x_{1}, x_{2}<1\right\}$.

We first consider the case that the support of $u_{*}$ is on $K \times\{0\}$ with $\frac{5}{4} K \times(0,1) \subset \Omega$. In this case we have $\int_{K} u_{*}^{3}=0$. Extend $u_{*}\left(x^{\prime}\right)=0$ for $x^{\prime} \in \mathbb{R}^{2} \backslash K$. Choose $\phi \in C^{\infty}\left(\mathbb{R}^{2}\right)$ so that $\phi(\xi)=\phi(|\xi|)$, $\phi(\xi)=0$ for $|\xi|<1 / 8$ and $|\xi|>1 / 4$, and $\int_{\mathbb{R}^{2}} \phi(\xi) d \xi=1$. Also choose $\chi \in C^{\infty}(\mathbb{R})$ so that $\chi(s)=1$ for $s<1 / 8$ and $\chi(s)=0$ for $s>1 / 4$. We define the extension by

$$
u(x)=\left(\partial_{3} \Phi_{1}, \quad \partial_{3} \Phi_{2}, \quad-\partial_{1} \Phi_{1}-\partial_{2} \Phi_{2}\right),
$$

where1

$$
\Phi_{j}\left(x^{\prime}, x_{3}\right)=\chi\left(x_{3}\right)\left[x_{3} \int_{\mathbb{R}^{2}} u^{j}\left(x^{\prime}+x_{3} \xi\right) \phi(\xi) d \xi+f^{j}\left(x^{\prime}\right)\right], \quad(j=1,2),
$$

and, with $g\left(x_{1}\right)=\int_{\mathbb{R}} u_{*}^{3}\left(x_{1}, \bar{x}_{2}\right) d \bar{x}_{2}$, supported in $\left|x_{1}\right| \leq 1$ and $\int_{-1}^{1} g\left(x_{1}\right) d x_{1}=0$,

$$
f^{1}\left(x^{\prime}\right)=-\chi^{\prime}\left(x_{2}\right) \int_{-\infty}^{x_{1}} g\left(\bar{x}_{1}\right) d \bar{x}_{1}, \quad f^{2}\left(x^{\prime}\right)=\int_{-\infty}^{x_{2}} u_{*}^{3}\left(x_{1}, \bar{x}_{2}\right) d \bar{x}_{2}-g\left(x_{1}\right)\left(1-\chi\left(x_{2}\right)\right) .
$$

(See the proof of Lemma 2.6.) Note $f^{j}$ are supported in $\bar{K}$, $\operatorname{div}_{\mathbb{R}^{2}} f=u_{*}^{3}$, and $u$ is supported in $\frac{9}{8} \bar{K} \times[0,1 / 4]$. One verifies (2.24) directly.

We next consider the case that the support of $u_{*}$ is on a graph over $K: x_{3}=h\left(x^{\prime}\right)$ for $x^{\prime} \in K$ with $\left|h\left(x^{\prime}\right)\right|<1 / 4$, and $\Omega \cap\left(\frac{3}{2} K \times[-1,1]\right)$ lies in $x_{3}>h\left(x^{\prime}\right)$. We have

$$
N=\frac{1}{\ell}\left(\partial_{1} h, \partial_{2} h,-1\right), \quad \ell=\sqrt{|\nabla h|^{2}+1}, \quad d A=\ell d x_{1} d x_{2},
$$

\footnotetext{
${ }^{1}$ The formula for $u^{3}$ is from Lemma 2.5. The formula for $u^{1}$ and $u^{2}$ extends the $n=2$ case of [15] (7.3)].
} 
and $0=\int_{\partial \Omega} u_{*} \cdot N d A=\int_{K} u_{*} \cdot\left(\partial_{1} h, \partial_{2} h,-1\right) d x_{1} d x_{2}$. Define new coordinates

$$
y_{1}=x_{1}, \quad y_{2}=x_{2}, \quad y_{3}=x_{3}-h\left(x^{\prime}\right),
$$

and, for each vector field defined for $x \in \bar{\Omega} \cap(K \times[0,1])$ define a new vector field for $y \in K \times$ $[-3 / 4,3 / 4]$ :

$$
U(y)=\left(u^{1}(x), \quad u^{2}(x), \quad u^{3}(x)-u^{1}(x) \partial_{1} h\left(x^{\prime}\right)-u^{2}(x) \partial_{2} h\left(x^{\prime}\right)\right) .
$$

For a given $u_{*}$ defined on $\partial \Omega$, we define $U_{*}$ on $K$ by the same formula. Then $U_{*}$ satisfies $\int_{K} U_{*}^{3}=0$ and we can extend $U$ from $U_{*}$ by the previous case so that $\operatorname{div} U=0$ and $\operatorname{supp} U \subset K \times[-1 / 2,1 / 2]$. We finally define $u$ from $U$ by (2.33). One checks directly that $\operatorname{div}_{x} u=\operatorname{div}_{y} U=0$.

For the general case, we can find finitely many balls $B_{j}, j=1, \ldots, J$, with same radius $\rho$ and concentric balls $B_{j}^{*}$ with double radius $2 \rho$, so that $\partial \Omega \subset \cup_{j} B_{j}, \cup_{j} B_{j}^{*} \subset B_{R_{1}}$, and that $\partial \Omega \cap B_{j}^{*}$ is a graph in $B_{j}^{*}$ in direction $\mu_{j}$, and belongs to $\left|\left(x-x_{j}\right) \cdot \mu_{j}\right| \leq \rho / 8$ where $x_{j}$ is the center of $B_{j}$. Choose a smooth partition of unity $\left\{\eta_{j}\right\}_{j}$ on $\partial \Omega$ so that $\sum_{j} \eta_{j}=1$ on $\partial \Omega$ and $\operatorname{supp} \eta_{j} \subset B_{j} \cap \partial \Omega$.

For any given $u_{*}$ with $\int_{\Gamma_{k}} u_{*} \cdot N=0$ for all $k$, we claim we can decompose

$$
u_{*}=\sum_{j} u_{*, j}, \quad \operatorname{supp} u_{*, j} \subset \partial \Omega \cap B_{j}, \quad \int_{\partial \Omega} u_{*, j} \cdot N=0,
$$

with suitable estimates. When $\partial \Omega$ has only one component $(M=1)$, choose $\chi_{k}, k=1, \ldots, J-1$, so that $\chi_{k} \in C_{c}^{\infty}\left(\partial \Omega \cap B_{k} \cap \cup_{j>k} B_{j}\right)$ with $\int_{\partial \Omega} \chi_{k}=1$. We define $u_{*, j}$ by induction: Let $U_{1}=u_{*}$, and for $j=1, \ldots, J-1$,

$$
u_{*, j}=U_{j} \eta_{j}-\chi_{j} \int_{\partial \Omega} U_{j} \eta_{j}, \quad U_{j+1}=U_{j}-u_{*, j}
$$

and $u_{*, J}=U_{J}$. When $\partial \Omega$ has more than one component, we can perform the above decomposition for each component. (The above decomposition follows the proof of [10, Lemma III.3.2].)

Since $\int_{\partial \Omega} u_{*, j} \cdot N=0$, by the second case above with a suitable rescaling we can extend $u_{*, j}$ to divergence-free $u_{j}$ supported in $B_{j}^{*} \cap \bar{\Omega}_{\delta}$. We now define $\mathcal{E}\left(u_{*}\right)=\sum_{j} u_{j}$.

Remark. If $u_{*}$ is prescribed in $K \times\{0,1\}$ with $u_{*}\left(x^{\prime}, 0\right)=\left(0,0, v_{0}\left(x^{\prime}\right)\right), u_{*}\left(x^{\prime}, 1\right)=\left(0,0, v_{1}\left(x^{\prime}\right)\right)$ and $\int_{K} v_{0}\left(x^{\prime}\right) d x^{\prime}=\int_{K} v_{1}\left(x^{\prime}\right) d x^{\prime}$, we can extend $u_{*}$ to $K \times[0,1]$ by

$$
u(x)=\left(\psi^{\prime}\left(x_{3}\right) f^{1}\left(x^{\prime}\right), \psi^{\prime}\left(x_{3}\right) f^{2}\left(x^{\prime}\right),\left(1-\psi\left(x_{3}\right)\right) v_{0}\left(x^{\prime}\right)+\psi\left(x_{3}\right) v_{1}\left(x^{\prime}\right)\right),
$$

where $\psi=1-\chi$

$$
f^{1}\left(x^{\prime}\right)=\psi^{\prime}\left(x_{2}\right) \int_{-\infty}^{x_{1}} g\left(\bar{x}_{1}\right) d \bar{x}_{1}, \quad f^{2}\left(x^{\prime}\right)=\int_{-\infty}^{x_{2}}\left(v_{0}-v_{1}\right)\left(x_{1}, \bar{x}_{2}\right) d \bar{x}_{2}-g\left(x_{1}\right) \psi\left(x_{2}\right)
$$

and $g\left(x_{1}\right)=\int_{\mathbb{R}}\left(v_{0}-v_{1}\right)\left(x_{1}, s\right) d s$.

Proof of Lemma 2.5. The case $M=1$ follows from Lemma 2.4, with $\delta>0$ chosen so small that $\Omega_{\delta} \subset \omega$. Suppose now $M \geq 2$.

Claim: We can choose $M-1$ line segments $L_{k}$ connecting $\Gamma_{j}$, so that $L_{k}$ intersect $\partial \Omega$ only at end points and at right angles, and each $\Gamma_{j}$ intersects at least one $L_{k}$. This is chosen by induction. Let $A_{1}=\{1\}$ and $A_{M}=\{1, \ldots, M\}$. Suppose $A_{k}$, a subset of $A_{M}$ has been chosen for $k=1, \ldots, M-1$. Since $\partial \Omega$ is compact, one can find a line segment $L_{k}$ minimizing the distance

$$
\operatorname{dist}\left(\cup_{j \in A_{k}} \Gamma_{j}, \cup_{j \notin A_{k}} \Gamma_{j}\right) \text {. }
$$

Clearly $L_{k}$ intersects $\cup_{j \in A_{k}} \Gamma_{j}$ only at one endpoint and $L_{k}$ intersects $\cup_{j \notin A_{k}} \Gamma_{j}$, say $\Gamma_{j k+1}$, at the other endpoint. Moreover, $L_{k}$ without endpoints is inside $\Omega$. Now let $A_{k+1}=A_{k} \cup\left\{j_{k+1}\right\}$ and continue.

Once all $L_{k}$ have been chosen, we give a rank of $\Gamma_{j}$ and $L_{k}$ as follows: $\Gamma_{j}$ is assigned rank one if it intersects only one $L_{k}$. This line segment is renumbered as $L_{j}$ and also assigned rank 1 . Let $A$ be the set of $\Gamma_{j}$ and $L_{k}$ without those of rank one. Rank one boundaries and line segments in this reduced set are assigned rank 2 . We continue this exercise until we exhaust all $\Gamma_{j}$ and $L_{k}$. 
Fix $0<\varepsilon \ll 1$. For each $k$, let $e_{k}$ be the unit direction vector of $L_{k}$ (unique up to a negative sign), let $L_{k}^{\varepsilon}$ be those points on the line extending $L_{k}$ with distance to $L_{k}$ less than $\varepsilon$, let $r_{k}(x)=\operatorname{dist}\left(x, L_{k}^{\varepsilon}\right)$, and let $U^{k}(x)=\varepsilon^{1-n} \chi\left(\varepsilon^{-1} r_{k}(x)\right) e_{k}$ where $\chi: \mathbb{R} \rightarrow[0,1]$ is a fixed smooth cut-off function with $\chi(r)=1$ for $r<1$ and $\chi(r)=0$ for $r>2$. For $\varepsilon>0$ sufficiently small (depending on $\partial \Omega$ ), we have $\int_{\Gamma_{j}} U^{k} \cdot N= \pm\left(\int_{\mathbb{R}^{n-1}} \chi\left(\left|x^{\prime}\right|\right) d x^{\prime}+o(1)\right) \neq 0$ if $\Gamma_{j}$ is one of the two boundaries intersecting $L_{k}$. We also have $\operatorname{supp} U^{k} \subset \omega$.

Now, for a given $u_{*}$, let $u_{*}^{0}=u_{*}$ and define recursively for $k \geq 1$ :

$$
u_{*}^{k}(x)=u_{*}^{k-1}(x)-\sum_{\Gamma_{j} \text { of rank } k} c_{j} U^{j}(x), \quad c_{j}=\frac{\int_{\Gamma_{j}} u_{*}^{k-1} \cdot N}{\int_{\Gamma_{j}} U^{j} \cdot N} .
$$

Denote the final one as $u_{*}^{M}$. One verifies that $\int_{\Gamma_{k}} u_{*}^{M} \cdot N=0$ for all $k$ and $\left\|u_{*}^{M}\right\|_{C^{l, \alpha}}+\sum_{j}\left|c_{j}\right| \leq$ $C\left\|u_{*}^{M}\right\|_{C^{l, \alpha}}$. We now define

$$
\mathcal{E}\left(u_{*}\right)=\mathcal{E}\left(u_{*}^{M}\right)+\sum_{j=1}^{M} c_{j} U^{j}(x),
$$

where $\mathcal{E}\left(u_{*}^{M}\right)$ is defined by Lemma 2.4. One verifies that $\left\|\mathcal{E}\left(u_{*}\right)\right\|_{C^{l, \alpha}(\Omega)} \leq C\left\|u_{*}^{M}\right\|_{C^{l, \alpha}}+C \sum_{j}\left|c_{j}\right| \leq$ $C\left\|u_{*}^{M}\right\|_{C^{l, \alpha}}$, and that the support of $\mathcal{E}\left(u_{*}\right)$ is inside $\omega$.

\subsection{Source terms}

In this subsection we show that any force $f(x)$ in $\mathbb{R}^{n}$ decaying like $|x|^{-n-\varepsilon}$ as infinity can be decomposed in the form $f=f_{0}+\nabla \cdot F$ with $\operatorname{supp} f_{0}$ being compact.

Lemma 2.6 If $f(x)$ is defined in $\mathbb{R}^{n}$ with $|f(x)| \lesssim\langle x\rangle^{-a}, a>n \geq 1$, then for any $R>0$ we can rewrite

$$
f(x)=f_{0}(x)+\sum_{j=1}^{n} \partial_{j} F_{j}(x)
$$

where supp $f_{0} \in B_{R}(0)$ and $\left|F_{j}(x)\right| \lesssim\langle x\rangle^{-a+1}\left\|\langle x\rangle^{a} f(x)\right\|_{L^{\infty}}$.

Remark. (i) If $f(x)$ is defined in an exterior domain $\Omega \subset \mathbb{R}^{n}$ with $0 \notin \bar{\Omega}$, we may extend $f$ by zero to entire $\mathbb{R}^{n}$, and choose $R>0$ so small that $\Omega \cap B_{R}=\emptyset$. Then the first term $f_{0}(x)$ in the decomposition (2.41) can be ignored for $x \in \Omega$.

(ii) If $f=g_{0}+\nabla \cdot G$ with $\left|g_{0}(x)\right| \lesssim\langle x\rangle^{-a}, a>n \geq 1$, and $|G(x)| \lesssim\langle x\rangle^{-a+1}$, we may assume $g_{0}$ has compact support by decomposing $g_{0 i}=f_{0 i}(x)+\sum_{j=1}^{n} \partial_{j} F_{j i}(x)$ as in the lemma and absorbing $F$ to $G$. If $f$ is defined in an exterior domain we may assume $g_{0}=0$ by (i) above.

Proof. We will prove the case $n=3$. The proof for the general case is similar. By rescaling we may assume $R=1$ and $\left\|\langle x\rangle^{a} f(x)\right\|_{L^{\infty}}=1$. We first consider the case $\operatorname{supp} f \subset B_{2}$. Choose a smooth $\psi(t): \mathbb{R} \rightarrow \mathbb{R}, \psi(t)=1$ for $t>R_{1}=3^{-1 / 2}$ and $\psi(t)=0$ for $t<-R_{1}$. The region $\left\{x:\left|x_{j}\right|<R_{1}\right\} \subset B_{1}$. Define

$$
\begin{gathered}
G_{3}(x)=\int_{-\infty}^{x_{3}} f\left(x_{1}, x_{2}, \bar{x}_{3}\right) d \bar{x}_{3}, \quad I_{3}\left(x_{1}, x_{2}\right)=\lim _{x_{3} \rightarrow+\infty} G_{3}(x), \\
F_{3}(x)=G_{3}(x)-\psi\left(x_{3}\right) I_{3}\left(x_{1}, x_{2}\right) .
\end{gathered}
$$

Then $f=\partial_{3} F_{3}+\psi^{\prime}\left(x_{3}\right) I_{3}\left(x_{1}, x_{2}\right)$. Define

$$
\begin{gathered}
G_{2}\left(x_{1}, x_{2}\right)=\int_{-\infty}^{x_{2}} I_{3}\left(x_{1}, \bar{x}_{2}\right) d \bar{x}_{2}, \quad I_{2}\left(x_{1}\right)=\lim _{x_{2} \rightarrow+\infty} G_{2}\left(x_{1}, x_{2}\right), \\
F_{2}(x)=\psi^{\prime}\left(x_{3}\right)\left[G_{2}\left(x_{1}, x_{2}\right)-\psi\left(x_{2}\right) I_{2}\left(x_{1}\right)\right] .
\end{gathered}
$$

Then $f=\partial_{3} F_{3}+\partial_{2} F_{2}+\psi^{\prime}\left(x_{2}\right) \psi^{\prime}\left(x_{3}\right) I_{2}\left(x_{1}\right)$. Define

$$
G_{1}\left(x_{1}\right)=\int_{-\infty}^{x_{1}} I_{2}\left(\bar{x}_{1}\right) d \bar{x}_{1}, \quad I=\lim _{x_{1} \rightarrow+\infty} G_{1}\left(x_{1}\right)=\int_{\mathbb{R}^{3}} f,
$$




$$
F_{1}(x)=\psi^{\prime}\left(x_{2}\right) \psi^{\prime}\left(x_{3}\right)\left[G_{1}\left(x_{1}\right)-\psi\left(x_{1}\right) I\right]
$$

Then

$$
f=\partial_{3} F_{3}+\partial_{2} F_{2}+\partial_{1} F_{1}+\psi^{\prime}\left(x_{1}\right) \psi^{\prime}\left(x_{2}\right) \psi^{\prime}\left(x_{3}\right) I .
$$

The last term vanishes if $\int f=0$. Also note $F_{j}$ are at least as regular as $f$,

$$
\left|F_{j}(x)\right| \lesssim\|f\|_{L^{\infty}}, \quad \text { and } \operatorname{supp} F_{j} \subset\left\{x:\left|x_{j}\right|<2\right\} .
$$

For the general case, choose a smooth $\varphi(x)$ supported in $\left\{x \in \mathbb{R}^{3}: 2^{-1} \leq|x| \leq 2\right\}$ satisfying $\varphi(x)>0$ for $2^{-1}<|x|<2$ and $\sum_{k=-\infty}^{\infty} \varphi\left(2^{-k} x\right)=1$ for $x \neq 0$. Let $\varphi_{k}(x)=\varphi\left(2^{-k} x\right)$ for $k \geq 0$ and $\psi(x)=1-\sum_{k=1}^{\infty} \varphi\left(2^{-k} x\right)$. Define

$$
a_{k}=\frac{1}{2^{k} \int \varphi} \int f \sum_{j>k} \varphi_{j}, \quad(k \geq 0) .
$$

Then $\left|a_{k}\right| \lesssim 2^{-k a}$. Decompose

$$
f=\sum_{k=0}^{\infty} f_{k}, \quad f_{0}=f \psi+a_{0} \varphi_{0}, \quad f_{k}=\left(f+a_{k}\right) \varphi_{k}-a_{k-1} \varphi_{k-1}, \quad(k \geq 1) .
$$

One verifies that

$$
\int f_{0}=\int f, \quad \int f_{k}=0, \quad(k \geq 1),
$$

supp $f_{k} \subset\left\{x: 2^{k-2} \leq|x| \leq 2^{k+1}\right\}$, and $\left|f_{k}(x)\right| \leq C\langle x\rangle^{-a}$. The rescaled function $\tilde{f}_{k}(x)=f_{k}\left(2^{k} x\right)$ is supported in $B_{2}$. The previous case gives the existence of $F_{k j}, j=1,2,3$, so that

$$
f_{k}=\sum_{j=1}^{3} \partial_{j} F_{k j}, \quad\left|F_{k j}(x)\right| \leq C\langle x\rangle^{-a+1}
$$

Thus

$$
f=f_{0}+\sum_{j=1}^{3} \partial_{j} F_{j}, \quad F_{j}=\sum_{k=1}^{\infty} F_{k j}, \quad\left|F_{j}(x)\right| \leq \sum_{k \gtrsim \ln |x|} 2^{-(a-1) k} \lesssim\langle x\rangle^{-a+1} .
$$

This shows the lemma.

\subsection{Stokes system}

In this subsection we proved decay estimates for the Stokes system in $\mathbb{R} \times \mathbb{R}^{3}$. Recall the fundamental solutions of the Stokes system (see [28] and [33, page 27])

$$
S_{i j}(t, x)=\Gamma(t, x) \delta_{i j}+\frac{1}{4 \pi} \frac{\partial^{2}}{\partial x_{i} \partial x_{j}} \int_{\mathbb{R}^{3}} \frac{\Gamma(t, y)}{|x-y|} d y, \quad Q_{j}(t, x)=\frac{\delta(t)}{4 \pi} \frac{x_{j}}{|x|^{3}},
$$

where $\Gamma$ is the fundamental solution of the heat equation. It is known in [33, Theorem 1] that the tensor $S=\left(S_{i j}\right)$ satisfies the following estimates:

$$
\left|D_{x}^{\ell} \partial_{t}^{k} S(t, x)\right| \leq C_{k, l}(|x|+\sqrt{t})^{-3-\ell-2 k}, \quad(\ell, k \geq 0),
$$

where $D_{x}^{\ell}$ indicates $\ell$-th order derivatives with respect to the variable $x$.

A solution of the non-stationary Stokes system in $\mathbb{R}^{3}$,

$$
\partial_{t} w-\Delta w+\nabla p=g+\nabla G, \quad \operatorname{div} w=0, \quad\left((t, x) \in \mathbb{R}^{1+3}\right),
$$

if $g$ and $G$ have sufficient decay, is given by

$$
w=\Lambda(g)+\Theta(G)
$$

where

$$
\begin{aligned}
& (\Lambda g)_{i}(t, x)=\int_{0}^{\infty} \int_{\mathbb{R}^{3}} S_{i j}(s, x-y) g_{j}(y, t-s) d y d s \\
& (\Theta G)_{i}(t, x)=-\int_{0}^{\infty} \int_{\mathbb{R}^{3}} \partial_{k} S_{i j}(s, x-y) G_{j k}(y, t-s) d y d s
\end{aligned}
$$


Lemma 2.7 (i) Suppose $G \in L^{\infty} X_{\alpha+1}, 0<\alpha<2$. Then $\Theta G \in L^{\infty} X_{\alpha}$ with $\|\Theta G\|_{L^{\infty} X_{\alpha}} \leq$ $C_{\alpha}\|G\|_{L^{\infty} X_{\alpha+1}}$.

(ii) Suppose $g \in L^{\infty} X_{\alpha+2}, 1<\alpha$. Then $\Lambda g \in L^{\infty} X_{1}$ with $\|\Lambda g\|_{L^{\infty} X_{1}} \leq C_{\alpha}\|g\|_{L^{\infty} X_{\alpha+2}}$.

(iii) Suppose $g \in L^{\infty} X_{\alpha+2}, 1<\alpha, g$ is time periodic of period $T>0$, and $\int_{0}^{T} \int g d x d t=0$. Then $\Lambda g \in L^{\infty} X_{2}$ with $\|\Lambda g\|_{L^{\infty} X_{2}} \leq C_{\alpha}\|g\|_{L^{\infty} X_{\alpha+2}}$.

Note that time periodicity is only assumed in (iii).

Proof. (i) For $\Theta G$ defined by (2.60), using the estimate (2.56) and integrating in time, we obtain

$$
|\Theta G(t, x)| \leq \int_{0}^{\infty} \int_{\mathbb{R}^{3}} \frac{C}{(|x-y|+\sqrt{s})^{4}}\langle y\rangle^{-\alpha-1} d y d s \lesssim I_{\alpha}(x),
$$

where

$$
I_{\alpha}(x)=\int_{\mathbb{R}^{3}} \frac{1}{|x-y|^{2}}\langle y\rangle^{-\alpha-1} d y, \quad(\alpha>0) .
$$

If $|x|<10$, then $I_{\alpha}(x) \lesssim 1$. If $|x| \geq 10$, then

$$
I_{\alpha}(x) \leq \int_{|y|<1} \frac{d y}{|x|^{2}}+\int \frac{d y}{|x-y|^{2}|y|^{\alpha+1}}=C|x|^{-2}+C|x|^{-\alpha},
$$

if $0<\alpha<2$. We conclude $I_{\alpha}(x) \leq C_{\alpha}\langle x\rangle^{-\alpha}$ and the estimate for $\Theta G$.

(ii) Due to Lemma 2.6 $g$ can be decomposed as $g=g_{0}+\nabla \tilde{G}$, where $\operatorname{supp} g_{0} \in B_{1}(0)$ and $\|\tilde{G}\|_{L^{\infty} X_{\alpha+1}} \lesssim\|g\|_{L^{\infty} X_{\alpha+2}}$. Since $\nabla \tilde{G}$ can be treated as in the case (i), we consider the only the case that $g_{0} \neq 0$ and $\tilde{G}=0$. We may assume $\left\|g_{0}\right\|_{L^{\infty} X_{\alpha+2}} \leq 1$. By Young's convolution inequality

$$
\begin{aligned}
|\Lambda g(t, x)| & \lesssim \int_{0}^{\infty}\left\|S_{i j}(s, \cdot)\right\|_{L^{2}+L^{\infty}}\left\|g_{j}(\cdot, t-s)\right\|_{L^{2} \cap L^{1}} d s \\
& \lesssim \int_{0}^{\infty} \min \left(s^{-3 / 4}, s^{-3 / 2}\right) d s \lesssim 1 .
\end{aligned}
$$

For $|x|>2$ we have

$$
\begin{aligned}
|\Lambda g(t, x)| & \leq \int_{0}^{\infty} \int_{|y| \leq 1}\left|S_{i j}(x-y, s)\right|\left|g_{j}(y, t-s)\right| d y d s \\
& \leq C \int_{0}^{\infty} \int_{|y| \leq 1} \frac{1}{(|x|+\sqrt{s})^{3}} d y d s \leq C \int_{0}^{\infty} \frac{1}{(|x|+\sqrt{s})^{3}} d s \leq \frac{C}{|x|} .
\end{aligned}
$$

(iii) Continue part (ii) and assume $|x|>2$. Using $\int_{0}^{T} \int g(t, y) d y d t=0$, we have

$$
(\Lambda g)_{i}(t, x)=\int_{0}^{\infty} \int_{\mathbb{R}^{3}}\left(S_{i j}(s, x-y)-S_{i j}(s, x)\right) g_{j}(y, t-s) d y d s .
$$

Using the mean-value formula and (2.56),

$$
\begin{aligned}
|(\Lambda g)(t, x)| & \leq \int_{0}^{\infty} \int_{|y| \leq 1}\left|\nabla_{y} S_{i j}(s, x-\theta y) \cdot y\right|\left|g_{j}(t-s, y)\right| d y d s \\
& \leq C \int_{0}^{\infty} \int_{|y| \leq 1} \frac{1}{(|x-\theta y|+\sqrt{s})^{4}} d y d s \leq C \int_{0}^{\infty} \frac{1}{(|x|+\sqrt{s})^{4}} d s \leq \frac{C}{|x|^{2}},
\end{aligned}
$$

where $\theta=\theta(x, y, s) \in[0,1]$, and we have used that $g_{j}$ is bounded and supported in $|y| \leq 1$ and $|x|>2$. This shows $\|\Lambda g\|_{L^{\infty} X_{2}} \leq C_{\alpha}\|g\|_{L^{\infty} X_{\alpha+2}}$ and completes the proof.

\section{Existence of flows with non-decaying boundary data}

In this section we prove Theorem 1.1. By Lemma 2.6. we may assume $f_{0}=0$ by absorbing $f_{0}$ into $F$. 


\subsection{Construction of a mild solution}

Proof of part (i). We first consider the case $I=\mathbb{R}$ and denote

$$
\mathcal{X}^{q}=L^{\infty}\left(\mathbb{R}, L^{q, \infty}\right)
$$

Let $\Gamma_{k}, k=1, \ldots, M$, be the connected components of $\partial \Omega$, and choose $x_{k}$ in the bounded open region enclosed by $\Gamma_{k}$. Let $H_{0}(x)=\frac{1}{|x|}$. Note

$$
\int_{\Gamma_{k}} \nabla H_{0}\left(x-x_{l}\right) \cdot N=4 \pi \delta_{k l}, \quad \forall k, l .
$$

For each $t \in I$, let

$$
H(t, x)=\sum g_{k}(t) H_{0}\left(x-x_{k}\right), \quad g_{k}(t)=\frac{1}{4 \pi} \int_{\Gamma_{k}} u_{*}(t) \cdot N
$$

Note $H$ is harmonic in $x \in \Omega$. Denote $u_{*}^{\prime \prime}(t)=u_{*}(t)-\left.\nabla H(t)\right|_{\partial \Omega}$. By (3.2) and (3.3),

$$
\int_{\Gamma_{k}} u_{*}^{\prime \prime}(t) \cdot N=0, \quad \forall k, \forall t
$$

By Lemma 2.4, we can define $E_{1}=\mathcal{E}\left(u_{*}^{\prime \prime}(t)\right) \in C^{2}(\bar{\Omega})$ with compact support in $B_{R_{1}}$.

Decompose

$$
u=v+E, \quad E=\nabla H+E_{1}, \quad p=\pi-\partial_{t} H .
$$

Then we have $\left.E\right|_{\partial \Omega}=u_{*}$, and $v, \pi$ satisfy

$$
v_{t}-\Delta v+\nabla \pi=g(v), \quad \operatorname{div} v=0,\left.\quad v\right|_{\partial \Omega}=0,
$$

where

$$
g(v)=\nabla \cdot[F-(E+v) \otimes(E+v)]-\partial_{t} E_{1}+\Delta E_{1}
$$

and $E$ satisfies $\|E\|_{L^{3 / 2, \infty}} \leq\left\|u_{*}\right\|_{C^{2}(\partial \Omega)}$. Moreover, since $\partial_{t} E_{1}$ is compactly supported in $\Omega$, we can apply Lemma 2.6 so that $\partial_{t} E_{1}$ can be written as

$$
\partial_{t} E_{1}=\nabla F_{1} \quad \text { with } \quad\left\|F_{1}\right\|_{L^{q, \infty}} \lesssim\left\|\langle\cdot\rangle^{a} \partial_{t} E_{1}\right\|_{L^{\infty}} \lesssim\left\|\partial_{t} u_{*}\right\|_{C^{2}(\partial \Omega)}
$$

for $q \geq 3 / 2$ and $a>3$. Therefore we can rewrite

$$
g(v)=\nabla G(v) \quad \text { with } \quad\|G(v)\|_{\mathcal{X}^{3 / 2}} \lesssim\|F\|_{\mathcal{X}^{3 / 2}}+\left(\left\|u_{*}\right\|+\|v\|_{\mathcal{X}^{3}}\right)^{2}+\left\|u_{*}\right\| .
$$

Here, $\left\|u_{*}\right\|=\left\|u_{*}\right\|_{W^{1, \infty}\left(\mathbb{R}, C^{2}(\partial \Omega)\right)}$, and we do not distinguish $\nabla$ and $\nabla \cdot$, since the difference does not play any roles in our argument.

Following 37, we consider the fixed point problem

$$
v=\Phi v, \quad(\Phi v)(t):=\int_{-\infty}^{t} e^{-(t-s) A} P \nabla G(v)(s) d s,
$$

where the integral is defined weakly in the sense of Lemma 2.3.

Applying Lemma 2.3. we have

$$
\|\Phi v\|_{\mathcal{X}^{3}} \lesssim\|G(v)\|_{\mathcal{X}^{3 / 2}} \lesssim\|F\|_{\mathcal{X}^{3 / 2}}+\left(\left\|u_{*}\right\|+\|v\|_{\mathcal{X}^{3}}\right)^{2}+\left\|u_{*}\right\|
$$

Similarly,

$$
\|\Phi v-\Phi \tilde{v}\|_{\mathcal{X}^{3}} \lesssim\left(\left\|u_{*}\right\|+\|v\|_{\mathcal{X}^{3}}+\|\tilde{v}\|_{\mathcal{X}^{3}}\right)\|v-\tilde{v}\|_{\mathcal{X}^{3}}
$$

Thus, if

$$
\varepsilon=\|F\|_{\mathcal{X}^{3 / 2}}+\left\|u_{*}\right\|
$$


is sufficiently small, there is a unique fixed point of $\Phi$ in the class

$$
\|v\|_{\mathcal{X}^{3}} \lesssim \varepsilon
$$

and $v \in B C_{w}\left(\mathbb{R}, L_{\sigma}^{3, \infty}\right)$ by Lemma 2.3

The case $I=(0, \infty)$ is proved similarly: We define $g_{k}(t)$ and $E_{1}(t, x)$ as above for $t \geq 0$, and decompose $u=v+E$ as in (3.5). The vector field $v(t, x)$ satisfies the initial condition

$$
v(0, x)=v_{0}(x):=u_{0}(x)-E(0, x) .
$$

It is a fixed point of $\Phi$ where

$$
\Phi v=e^{-t A} v_{0}+\int_{0}^{t} e^{-(t-s) A} P \nabla G(v)(s) d s .
$$

Denote $\mathcal{X}_{+}^{q}=L^{\infty}\left(\mathbb{R}_{+}, L^{q, \infty}\right)$. By similar estimates, there is a unique fixed point of $\Phi$ in the class

$$
\|v\|_{\mathcal{X}_{+}^{3}} \leq C \varepsilon, \quad \varepsilon=\left\|v_{0}\right\|_{L^{3, \infty}}+\|F\|_{\mathcal{X}_{+}^{3 / 2}}+\left\|u_{*}\right\|,
$$

if $\varepsilon$ is sufficiently small, and $v \in B C_{w}\left([0, \infty), L_{\sigma}^{3, \infty}\right)$ by Lemma 2.3 .

\subsection{Equivalence to a very weak solution}

We now verify that our solution $u=v+E$ is the unique very weak solution in the class $u \in$ $B C_{w}\left(\bar{I}, L^{3, \infty}(\Omega)\right)$ of (1.1) with the given data $u_{0}, u_{*}$, and $f$, with $I=\mathbb{R}$ or $I=(0, \infty)$.

A mild solution is a very weak solution. We first show that our solution is a very weak solution. Clearly $u$ satisfies (2.22) and $\operatorname{div} u=0$. It suffices to show (2.21).

By divergence theorem, for $w \in \mathcal{D}_{I}$, defined in (2.20), and fixed $t$,

$$
\left(u_{*}, N \cdot \nabla w\right)_{\partial \Omega}=(E, N \cdot \nabla w)_{\partial \Omega}=(E, \Delta w)+(\nabla E, \nabla w)=(E, \Delta w)-(\Delta E, w) .
$$

Thus, upon writing $u=E+v,(2.21)$ is equivalent to

$$
\int_{I}-\left(v, w_{t}+\Delta w\right) d t=\left(v_{0}, w(0)\right)-\int_{I}\left(F_{2}, \nabla w\right) d t, \quad \forall w \in \mathcal{D}_{I}
$$

for $F_{2}=F_{2}(v)=F-(E+v) \otimes(E+v)+\nabla E_{1}-F_{1}$. Recall $\nabla F_{1}=\partial_{t} E_{1}$. Above $v_{0}=0$ if $I=\mathbb{R}$, and $v_{0}$ is given by (3.13) if $I=(0, \infty)$.

Let

$$
\mathcal{D}:=\left\{\psi \in C_{c}^{2}\left(\bar{\Omega} ; \mathbb{R}^{3}\right), \operatorname{div} \psi=0,\left.\psi\right|_{\partial \Omega}=0\right\} .
$$

Note $\left.\nabla \psi\right|_{\partial \Omega}$ may not be zero for $\psi \in \mathcal{D}$, and $\mathcal{D}$ is a dense subset of $D(A)$. Choosing $w(t, x)=\theta(t) \psi(x)$ with $\theta(t) \in C_{c}^{1}(\bar{I})$ and $\psi \in \mathcal{D}$, (2.21) implies

$$
\int_{I}-(v, \psi) \theta^{\prime} d t=\left(v_{0}, \psi\right) \theta(0)+\int_{I} \theta\left[\langle v, \Delta \psi\rangle-\left(F_{2}, \nabla \psi\right)\right] d t .
$$

In turn this also implies (2.21) since linear combinations of such $w(t, x)=\theta(t) \psi(x)$ with $\theta \in C_{c}^{1}(\bar{I})$ and $\psi \in \mathcal{D}$ is dense in $\mathcal{D}_{I}$ in the norm $\sum_{a \leq 1, b \leq 2}\left\|\partial_{t}^{a} \nabla_{x}^{b} w\right\|_{C^{0}}$.

Denote $t_{0}=\inf I$. Being a mild solution $v=\Phi v$ of (3.8) means $v(t)$ satisfies

$$
(v(t), \psi)=\left(e^{-t A} v_{0}, \psi\right)+\int_{t_{0}}^{t}\left(-F_{2}(s), \nabla e^{-(t-s) A} \psi\right) d s, \quad \forall \psi \in L_{\sigma}^{3 / 2,1}(\Omega), \forall t \in I .
$$

Plug this in the left side of (3.19). For $\psi \in \mathcal{D}$ and $\theta(t) \in C_{c}^{2}\left(\left(t_{0}, \infty\right)\right)$, since $\left[\theta^{\prime}(t)-\varepsilon^{-1}(\theta(t)-\theta(t-\right.$ $\varepsilon))] \rightarrow 0$ as $\varepsilon \rightarrow 0_{+}$uniformly in $t$,

$$
\begin{gathered}
\int_{I}-(v, \psi) \theta^{\prime} d t=\lim _{\varepsilon \rightarrow 0+} \varepsilon^{-1} \int_{I}(v(t), \psi)[-\theta(t)+\theta(t-\varepsilon)] d t= \\
=\lim _{\varepsilon \rightarrow 0+} \varepsilon^{-1} \int_{I}(v(t+\varepsilon)-v(t), \psi) \theta(t) d t=I_{\varepsilon}+I I_{\varepsilon}
\end{gathered}
$$


where

$$
\begin{aligned}
I_{\varepsilon}= & \int \theta(t)\left(e^{-t A} v_{0}, \varepsilon^{-1}\left(e^{-\varepsilon A}-1\right) \psi\right) d t \\
& +\int \theta(t) \int_{t_{0}}^{t}\left(-F_{2}(s), \nabla e^{-(t-s) A} \varepsilon^{-1}\left(e^{-\varepsilon A}-1\right) \psi\right) d s d t, \\
& I I_{\varepsilon}=\varepsilon^{-1} \int \theta(t) \int_{t}^{t+\varepsilon}\left(-F_{2}(s), \nabla e^{-(t+\varepsilon-s) A} \psi\right) d s d t .
\end{aligned}
$$

For $\psi \in \mathcal{D} \subset D(A),-A \psi=P \Delta \psi \in L_{\sigma}^{3 / 2,1}$ and

$$
\left\|\varepsilon^{-1}\left(e^{-\varepsilon A}-1\right) \psi+A \psi\right\|_{L_{\sigma}^{3 / 2,1}} \rightarrow 0 \quad \text { as } \quad \varepsilon \rightarrow 0_{+} .
$$

Thus, by Lemma 2.2 and $v \in B C_{w}\left(\bar{I} ; L_{\sigma}^{3, \infty}\right)$,

$$
\begin{aligned}
I_{\varepsilon} \rightarrow & \int \theta(t)\left(e^{-t A} v_{0}, P \Delta \psi\right) d t+\int \theta(t) \int_{t_{0}}^{t}\left(-F_{2}(s), \nabla e^{-(t-s) A} P \Delta \psi\right) d s d t \\
& =\int \theta(t)(v(t), P \Delta \psi) d t=\int \theta(t)(v(t), \Delta \psi) d t
\end{aligned}
$$

as $\varepsilon \rightarrow 0+$. Moreover, with $\tau=(t+\varepsilon-s)$ and by Fubini theorem,

$$
\begin{aligned}
I I_{\varepsilon} & =\varepsilon^{-1} \int \theta(t) \int_{0}^{\varepsilon}\left(-F_{2}(t+\varepsilon-\tau), \nabla e^{-\tau A} \psi\right) d \tau d t \\
& =-\varepsilon^{-1} \int_{0}^{\varepsilon}\left(\int \theta(t) F_{2}(t+\varepsilon-\tau) d t, \nabla e^{-\tau A} \psi\right) d \tau
\end{aligned}
$$

Since $\int \theta(t) F_{2}(t+\varepsilon-\tau) d t=\int \theta(t-\varepsilon+\tau) F_{2}(t) d t$ and $\theta(t-\varepsilon+\tau)-\theta(t) \rightarrow 0$ as $\varepsilon \rightarrow 0$ uniformly in $t$ and $\tau \in[0, \varepsilon]$, we get

$$
I I_{\varepsilon}+\varepsilon^{-1} \int_{0}^{\varepsilon}\left(\int \theta(t) F_{2}(t) d t, \nabla e^{-\tau A} \psi\right) d \tau \rightarrow 0
$$

as $\varepsilon \rightarrow 0_{+}$. Since $e^{-\tau A} \psi$ is continuous in $L_{\sigma}^{3 / 2,1}$, we get

$$
\lim _{\varepsilon \rightarrow 0_{+}} I I_{\varepsilon}=-\left(\int \theta(t) F_{2}(t) d t, \nabla \psi\right)
$$

The above shows (3.19) for $\psi \in \mathcal{D}$ and $\theta(t) \in C_{c}^{2}\left(\left(t_{0}, \infty\right)\right)$. By approximation, 3.19) is also valid for $\psi \in \mathcal{D}$ and $\theta(t) \in C_{c}^{1}\left(\left[t_{0}, \infty\right)\right)$.

A very weak solution is a mild solution. We next show that if $u$ is a small very weak solution in the class $B C_{w}\left(\bar{I}, L^{3, \infty}\right)$, then $v=u-E$ is a mild solution. This implies their equivalence, and also the uniqueness of small very weak solutions in the above class.

Let $u$ be small very weak solution in the class $B C_{w}\left(\bar{I}, L^{3, \infty}\right)$, then $v=u-E$ satisfies (3.19). Taking $\theta(t) \in C_{c}^{1}(I)$, (3.19) is the weak form of

$$
\frac{d}{d t}(v(t), \psi)=(v, \Delta \psi)-\left(F_{2}, \nabla \psi\right)
$$

If we now take $\psi=\psi(t)=e^{-\left(t_{1}-t\right) A} \eta$ with $t \leq t_{1}$ and $\eta \in \mathcal{D}$, we get $\frac{d}{d t} \psi(t)=-\Delta \psi(t)$ and $\frac{d}{d t}\left(v(t), e^{-\left(t_{1}-t\right) A} \eta\right)=-\left(F_{2}, \nabla e^{-\left(t_{1}-t\right) A} \eta\right)$ weakly, that is

$$
-\int\left(v(t), e^{-\left(t_{1}-t\right) A} \eta\right) \theta^{\prime}(t) d t=-\int\left(F_{2}(t), \nabla e^{-\left(t_{1}-t\right) A} \eta\right) \theta(t) d t
$$

for any $\theta(t) \in C_{c}^{1}\left(\left(\inf I, t_{1}\right)\right)$. Take $t_{0} \in\left[\inf I, t_{1}\right)$ and $\theta(t)=\phi\left(\frac{t-t_{1}}{\varepsilon}+1\right)-\phi\left(\frac{t-t_{0}}{\varepsilon}\right)$ where $0<\varepsilon \ll 1$, $\phi(t) \in C^{1}(\mathbb{R}), \phi(t)=1$ for $t<0$ and $\phi(t)=0$ for $t>1$. Send $\varepsilon \rightarrow 0_{+}$. We have $\theta(t) \rightarrow 1_{t_{0}<t<t_{1}}$ and, by continuity of $\left(v(t), e^{-\left(t_{1}-t\right) A} \eta\right)$,

$$
\left(v\left(t_{1}\right), \eta\right)-\left(v\left(t_{0}\right), e^{-\left(t_{1}-t_{0}\right) A} \eta\right)=-\int_{t_{0}}^{t_{1}}\left(F_{2}(t), \nabla e^{-\left(t_{1}-t\right) A} \eta\right) d t
$$


If $I=\mathbb{R}$, we send $t_{0} \rightarrow-\infty$. Since $v \in L^{\infty}\left(\mathbb{R} ; L^{3, \infty}\right)$ and $e^{-\left(t_{1}-t_{0}\right) A} \eta \rightarrow 0$ in $L_{\sigma}^{3 / 2,1}$ as $t_{0} \rightarrow-\infty$, we get

$$
\left(v\left(t_{1}\right), \eta\right)=-\int_{-\infty}^{t_{1}}\left(F_{2}(t), \nabla e^{-\left(t_{1}-t\right) A} \eta\right) d t, \quad \forall t_{1} \in I, \quad \forall \eta \in \mathcal{D} .
$$

If $I=(0, \infty)$, we take $t_{0} \rightarrow 0_{+}$and get

$$
\left(v\left(t_{1}\right), \eta\right)-\left(v_{0}, e^{-t_{1} A} \eta\right)=-\int_{0}^{t_{1}}\left(F_{2}(t), \nabla e^{-\left(t_{1}-t\right) A} \eta\right) d t, \quad \forall t_{1} \in I, \forall \eta \in \mathcal{D}
$$

In either case $v(t)$ is a mild solution and is the unique one we constructed in the previous subsection.

\subsection{Periodicity and spatial decay}

Proof of part (ii). Since $u(t+T, x)$ is another solution of (1.1)-(1.2) with the same data and estimates, we have $u(t+T, x)=u(t, x)$ by the uniqueness of part (i).

Proof of part (iii). We now assume $I=\mathbb{R}$ and the stronger assumption of (iii).

We first prove some a priori bounds. By Lemma 2.3 again for a fixed $q \in(3 / 2,3)$ and $q^{*}$, we have

$$
\begin{aligned}
\|v\|_{\mathcal{X}^{q^{*}}} & \lesssim\|G(v)\|_{\mathcal{X}^{q}} \\
& \lesssim\|F\|_{\mathcal{X}^{q}}+\left(\|E\|_{\mathcal{X}^{3}}+\|v\|_{\mathcal{X}^{3}}\right)\left(\|E\|_{\mathcal{X}^{q^{*}}}+\|v\|_{\mathcal{X}^{q^{*}}}\right)+\left\|u_{*}\right\| .
\end{aligned}
$$

Hence, we obtain,

$$
\|v\|_{\mathcal{X}^{q^{*}}} \lesssim\|F\|_{\mathcal{X}^{q}}+\left\|u_{*}\right\|
$$

when $\varepsilon$ is sufficiently small.

We now estimate $w=v_{t}$. It satisfies

$$
w_{t}-\Delta w+\nabla \pi_{t}=\nabla \partial_{t} G, \quad \operatorname{div} w=0,\left.\quad w\right|_{\partial \Omega}=0,
$$

where

$$
\partial_{t} G=\left(F_{t}-\left(E_{t}+w\right) \otimes(E+v)-(E+v) \otimes\left(E_{t}+w\right)\right)-\partial_{t}^{2} F_{1}+\partial_{t} \Delta E_{1} .
$$

For fixed $3 / 2<q<3$ we have

$$
\left\|\partial_{t} E\right\|_{\mathcal{X}^{q^{*}} \cap \mathcal{X}^{3}}+\left\|\partial_{t}^{2} F_{1}\right\|_{\mathcal{X}^{q} \cap \mathcal{X}^{3 / 2}}+\left\|\partial_{t} \nabla E_{1}\right\|_{\mathcal{X}^{q} \cap \mathcal{X}^{3 / 2}} \lesssim\left\|\partial_{t} u_{*}\right\|_{W^{1, \infty}\left(\mathbb{R}, C^{2}(\partial \Omega)\right)} .
$$

Similar estimates show $v_{t} \in \mathcal{X}^{3} \cap \mathcal{X}^{q^{*}}$ and

$$
\left\|v_{t}\right\|_{\mathcal{X}^{3} \cap \mathcal{X}^{q^{*}}} \lesssim \varepsilon_{1}:=\|F\|_{W^{1, \infty}\left(\mathbb{R}, L^{3 / 2} \cap L^{q}\right)}+\left\|u_{*}\right\|_{W^{2, \infty}\left(\mathbb{R}, C^{2}(\partial \Omega)\right)} .
$$

From now on we choose $q=2$ and thus $q^{*}=6$ for convenience.

Rewrite equation (3.6) for $v$ as a time-independent Stokes system,

$$
-\Delta v+\nabla \pi=g(v)-v_{t}, \quad \operatorname{div} v=0 .
$$

We have $v \in \mathcal{X}^{3} \cap \mathcal{X}^{q^{*}}, F \in \mathcal{X}^{3 / 2} \cap \mathcal{X}^{q}$, and $-\partial_{t} E_{1}+\Delta E_{1}-v_{t} \in \mathcal{X}^{3} \cap \mathcal{X}^{q^{*}}$. By bootstrapping as in [16], also see 25], locally $E$ and $\pi-\phi(t)$ with a suitable $\phi(t)$ are bounded uniformly in $t$. Let

$$
\mathcal{R}_{\text {cut }}:=\left\{x \in \mathbb{R}^{3}: \quad R_{1}<|x|<R_{1}+1\right\} .
$$

We now replace $\pi(t, x)$ by $\pi(t, x)-\phi(t)$ with a suitable $\phi(t)$ so that

$$
|v(t, x)|+|\nabla v(t, x)|+|\pi(t, x)| \leq C \varepsilon_{1}, \quad \forall t \in \mathbb{R}, \quad \forall x \in \mathcal{R}_{c u t} .
$$

Back to $u=E+v$ and $p=\pi-\partial_{t} H$, we have

$$
\begin{gathered}
\|u\|_{\mathcal{X}^{3} \cap \mathcal{X} q^{*}} \leq C \tilde{\varepsilon} ; \\
|u(t, x)|+|\nabla u(t, x)|+|p(t, x)| \leq C \tilde{\varepsilon}, \quad \forall t \in \mathbb{R}, \quad \forall x \in \mathcal{R}_{\text {cut }} .
\end{gathered}
$$

The pointwise decay estimate of (iii) follows from the following lemma. 
Lemma 3.1 (Spatial decay of time-entire solutions) For any $R_{1}>0$, there are $\varepsilon_{1}>0$ and $C>0$ such that the following holds. Suppose $u, p$ is a solution of (1.1) with force $f=f_{0}+\nabla \cdot F$ for $R_{1}<|x|<\infty$ and $t \in \mathbb{R}$, and satisfies

$$
\begin{aligned}
\varepsilon:= & \sup _{t \in \mathbb{R}}\left\{\sup _{|x|>R_{1}}\left\{|x|^{2+\alpha}\left|f_{0}(t, x)\right|+|x|^{1+\alpha}|F(t, x)|\right\}+\|u(t, \cdot)\|_{L^{3, \infty}\left(|x|>R_{1}\right)}+\right. \\
& \left.+\sup _{R_{1}<|x|<R_{1}+1}\{|u(t, x)|+|\nabla u(t, x)|+|p(t, x)|\}\right\} \leq \varepsilon_{1} .
\end{aligned}
$$

We do not assume any boundary condition at $|x|=R_{1}$. Then

$$
|u(t, x)| \leq C \varepsilon|x|^{-1}, \quad\left(|x|>R_{1} ; t \in \mathbb{R}\right) .
$$

Proof. We perform a cut-off and the extend the solution for $|x|>R_{1}$ to entire $\mathbb{R}^{3}$. Fix a smooth function $\zeta(x)$ which is 1 for $|x|>R_{1}+0.9$ and 0 for $|x|<R_{1}+0.1$. Let

$$
\tilde{u}=\zeta u+\nabla \eta, \quad \operatorname{div} \tilde{u}=0, \quad \tilde{p}=\zeta p-\partial_{t} \eta
$$

where, for each $t, \eta$ is defined by the Newtonian potential so that $\operatorname{div} \tilde{u}=0$, i.e.,

$$
\eta(t, x)=\int_{\mathcal{R}_{\text {cut }}} \frac{u(t, y) \cdot \nabla \zeta(y)}{4 \pi|x-y|} d y, \quad-\Delta \eta=u \cdot \nabla \zeta .
$$

Note that $\tilde{u}, \tilde{p}$ are defined for $x \in \mathbb{R}^{3}$ and satisfy the Stokes system for $(t, x) \in \mathbb{R}^{1+3}$

$$
\partial_{t} \tilde{u}-\Delta \tilde{u}+\nabla \tilde{p}=\zeta f_{0}+\nabla \cdot F_{3}(\tilde{u})+f_{4}, \quad \operatorname{div} \tilde{u}=0
$$

whert 2

$$
\begin{aligned}
F_{3}(\tilde{u})= & \zeta F-(\tilde{u}-\nabla \eta) \otimes(\tilde{u}-\nabla \eta), \\
f_{4}=- & \left.(\nabla \zeta) \cdot F+\nabla(u \cdot \nabla \zeta)+\nabla \cdot\left[\left(\zeta^{2}-\zeta\right) u \otimes u\right)\right] \\
& -2(\nabla \zeta \cdot \nabla) u-u \Delta \zeta+(u \cdot \nabla \zeta) u+p \nabla \zeta .
\end{aligned}
$$

Note $F_{3}$ contains $\tilde{u}$ and global source terms, while $f_{4}(t, x)$ contains only local source terms,

$$
\operatorname{supp} f_{4} \subset \mathbb{R} \times \mathcal{R}_{c u t}, \quad\left\|f_{4}\right\|_{L_{t, x}^{\infty}} \leq C \varepsilon_{1} .
$$

Consider now the fixed point problem $w=\Phi w$ for the map $\Phi$ from the class of vector fields defined on $\mathbb{R} \times \mathbb{R}^{3}$ into itself, defined by

$$
(\Phi w)=\Lambda\left(\zeta f_{0}+f_{4}\right)+\Theta\left(F_{3}(w)\right) .
$$

Here $\Lambda$ and $\Theta$ are defined by (2.59) and (2.60), respectively. We want to show it is a contraction mapping in the class of small vector fields in $L^{\infty} X_{1}$. By Lemma 2.7

$$
\begin{aligned}
\|\Phi w\|_{L^{\infty} X_{1}} & \leq C\left\|\zeta f_{0}+f_{4}\right\|_{L^{\infty} X_{3+\delta}}+C\left\|F_{3}\right\|_{L^{\infty} X_{2}} \\
& \leq C \varepsilon_{1}+C\left(\varepsilon_{1}+\|w\|_{L^{\infty} X_{1}}\right)\|w\|_{L^{\infty} X_{1}} \\
\|\Phi w-\Phi \tilde{w}\|_{L^{\infty} X_{1}} & \leq C\left(\varepsilon_{1}+\|w\|_{L^{\infty} X_{1}}+\|\tilde{w}\|_{L^{\infty} X_{1}}\right)\|w-\tilde{w}\|_{L^{\infty} X_{1}} .
\end{aligned}
$$

Thus there is a constant $C_{1}$ so that $\Phi$ is a contraction mapping in the class

$$
\left\{w(t, x): \mathbb{R} \times \mathbb{R}^{3} \rightarrow \mathbb{R}^{3}, \quad\|w\|_{L^{\infty} X_{1}} \leq C_{1} \varepsilon_{1}\right\} .
$$

Thus there is a unique solution of $w=\Phi w$ in $L^{\infty} X_{1}$.

By uniqueness of small solutions in $L^{\infty}\left(\mathbb{R}, L^{3, \infty}\left(\mathbb{R}^{3}\right)\right)$, we have $w=\tilde{u}=\zeta u+\nabla \eta$. Thus

$$
|u(t, x)| \leq|w(t, x)|+|\nabla \eta(t, x)| \leq C_{1} \varepsilon \frac{1}{|x|}, \quad\left(|x|>R_{1}+1\right) .
$$

\footnotetext{
${ }^{2}$ Eq. 3.52 seems complicated because it is used for the construction of $\tilde{u}$ without assuming decay estimates of $u$, and hence cannot allow $u$ in $F_{3}$.
} 


\section{Spatial asymptotics of time periodic solutions}

In this section we prove Theorem 1.2 We start with a $T$-periodic solution $(u, p)$ of (1.1)-(1.2) with force $F$ for $|x|>R$, satisfying the estimates assumed in Theorem 1.2 Proceed as in the proof of Lemma 3.1 and let $R_{1}=R$, we fix a cut-off function $\zeta(x)$ with $\nabla \zeta$ supported in $\mathcal{R}_{\text {cut }}$ and define

$$
\tilde{u}=\zeta u+\nabla \eta, \quad \tilde{p}=p \zeta-\partial_{t} \eta, \quad-\Delta \eta=\nabla \zeta \cdot u,
$$

as in (3.49) and (3.50). Then $\tilde{u}$ and $\tilde{p}$ satisfies (3.51)-3.53). By Lemma 3.1 we have $|u(t, x)| \leq$ $C \varepsilon|x|^{-1}$ for $|x|>R$.

Let $b$ be the constant vector defined by (1.14),

$$
b_{j}=\lim _{\rho \rightarrow \infty} \frac{1}{T} \int_{0}^{T} \int_{|x|=\rho} T_{i j}(u, p, F) n_{i} d S_{x} d t, \quad\left(n_{i}=\frac{x_{i}}{|x|}, i=1,2,3\right),
$$

where $T_{i j}(u, p, F)=p \delta_{i j}+u_{i} u_{j}-\partial_{i} u_{j}-\partial_{j} u_{i}-F_{i j}$. By the fast spatial decay of $\nabla \eta$, the time periodicity, and divergence theorem,

$$
b_{j}=\lim _{\rho \rightarrow \infty} \frac{1}{T} \int_{0}^{T} \int_{|x|=\rho} T_{i j}(\tilde{u}, \tilde{p}, F) n_{i} d S_{x} d t=\frac{1}{T} \int_{0}^{T} \int_{|x| \leq R_{1}+1}\left(\zeta f_{0}+f_{4}\right)_{j} d x d t .
$$

Also let $U^{b}$ be the corresponding Landau solution, and let

$$
\tilde{U}=\zeta U^{b}+\hat{U}, \quad \tilde{P}=\zeta P^{b},
$$

where $\hat{U}$ has compact support in $\mathcal{R}_{c u t}$, $\operatorname{div} \hat{U}=-\tilde{U} \cdot \nabla \zeta$, and

$$
\|\hat{U}\|_{W^{3,6}} \leq C\|\tilde{U} \cdot \nabla \zeta\|_{W^{2,6}\left(\mathcal{R}_{\text {cut }}\right)} \leq C|b| \leq C \varepsilon_{1} .
$$

Note that $\tilde{U}, \tilde{P}$ satisfies

$$
-\Delta \tilde{U}+\nabla \cdot(\tilde{U} \otimes \tilde{U})+\nabla \tilde{P}=f_{\tilde{U}}, \quad \operatorname{div} \tilde{U}=0,
$$

for $x \in \mathbb{R}^{3}$, where

$$
\begin{gathered}
f_{\tilde{U}}=-\Delta \hat{U}-2 \nabla U^{b} \cdot \nabla \zeta-U^{b} \Delta \zeta+P \nabla \zeta+\left(\zeta^{2}-\zeta\right) \nabla \cdot\left(U^{b} \otimes U^{b}\right) \\
+\nabla \cdot\left(\tilde{U} \otimes \tilde{U}-\zeta U^{b} \otimes \zeta U^{b}\right)+\left(U^{b} \cdot \nabla \zeta^{2}\right) U^{b} . \\
\left\|f_{\tilde{U}}\right\|_{L^{\infty}} \leq C \varepsilon_{1}, \quad \operatorname{supp} f_{\tilde{U}} \subset \mathcal{R}_{c u t} .
\end{gathered}
$$

Moreover,

$$
\begin{aligned}
\int_{\mathbb{R}^{3}}\left(f_{\tilde{U}}\right)_{j} d x & =\int_{|x| \leq R_{1}+2} \partial_{i} T_{i j}(\tilde{U}, \tilde{P}) d x=\int_{|x|=R_{1}+2} T_{i j}(\tilde{U}, \tilde{P}) \frac{x_{i}}{|x|} d S_{x} \\
& =\int_{|x|=R_{1}+2} T_{i j}\left(U^{b}, P^{b}\right) \frac{x_{i}}{|x|} d S_{x}=b_{j} .
\end{aligned}
$$

Let

$$
v=\tilde{u}-\tilde{U}, \quad \pi=\tilde{p}-\tilde{P}
$$

They satisfy, for $(t, x) \in \mathbb{R}^{1+3}$,

$$
\partial_{t} v-\Delta v+\nabla \pi=g+\nabla G(v), \quad \operatorname{div} v=0, \quad|v(t, x)| \leq C \varepsilon_{2}\langle x\rangle^{-1}
$$

where $\left(\right.$ note $\left.G(v)=F_{3}(\tilde{u})+\tilde{U} \otimes \tilde{U}\right)$

$$
\begin{aligned}
g & =\zeta f_{0}+f_{4}-f_{\tilde{U}}, \\
G(v) & =\zeta F-(v-\nabla \eta) \otimes(v+\tilde{U}-\nabla \eta)-\tilde{U} \otimes(v-\nabla \eta) .
\end{aligned}
$$

Because $\int_{\mathbb{R}^{3}}\left(f_{\tilde{U}}\right)_{i} d x=b_{i}$,

$$
\frac{1}{T} \int_{0}^{T} \int_{\mathbb{R}^{3}} g(t, x) d x d t=0 .
$$


Consider now the fixed point problem $v=\Phi v$ for the map $\Phi$ from the class of vector fields defined on $\mathbb{R} \times \mathbb{R}^{3}$ into itself, defined by

$$
\Phi v=\Lambda g+\Theta G(v) \text {. }
$$

Here $\Lambda$ and $\Theta$ are defined by (2.59) and (2.60), respectively. We want to show it is a contraction mapping in the class of small vector fields in $L^{\infty} X_{\alpha}, 1<\alpha<2$. By Lemma 2.7.

$$
\begin{aligned}
\|\Phi v\|_{L^{\infty} X_{\alpha}} & \leq C\|g\|_{L^{\infty} X_{3+\delta}}+C\|G(v)\|_{L^{\infty} X_{\alpha+1}} \\
& \leq C \varepsilon_{1}+C\|\zeta F\|_{X_{\alpha}}+C\|v-\nabla \eta\|_{X_{\alpha}}\left(\|v-\nabla \eta\|_{X_{\alpha}}+\|\tilde{U}\|_{X_{1}}\right) \\
& \leq C \varepsilon_{1}+C\left(\varepsilon_{1}+\|v\|_{L^{\infty} X_{\alpha}}\right)\|v\|_{L^{\infty} X_{\alpha}} \\
\|\Phi v-\Phi \tilde{v}\|_{L^{\infty} X_{\alpha}} & \leq C\left(\varepsilon_{1}+\|v\|_{L^{\infty} X_{\alpha}}+\|\tilde{v}\|_{L^{\infty} X_{\alpha}}\right)\|v-\tilde{v}\|_{L^{\infty} X_{\alpha}} .
\end{aligned}
$$

Thus there is a constant $C_{2}$ so that $\Phi$ is a contraction mapping in the class

$$
\left\{v(t, x): \mathbb{R} \times \mathbb{R}^{3} \rightarrow \mathbb{R}^{3}, \quad\|v\|_{L^{\infty} X_{\alpha}} \leq C_{2} \varepsilon_{1}\right\} .
$$

Thus there is a unique solution of $v=\Phi v$ in $L^{\infty} X_{\alpha}$, which agrees with $\tilde{u}-\tilde{U}$ by uniqueness of small solutions in $L^{\infty}\left(\mathbb{R}, L^{3, \infty}\left(\mathbb{R}^{3}\right)\right)$.

Since $v=\zeta u+\nabla \eta-\zeta U^{b}-\hat{U}$, for $|x|>R_{1}+1$, we have

$$
\left|u(t, x)+\nabla \eta(t, x)-U^{b}(x)\right| \leq C_{2} \varepsilon\langle x\rangle^{-\alpha} .
$$

Since $|\nabla \eta(t, x)| \leq C\langle x\rangle^{-2}$, we have proven Theorem 1.2

\section{Perturbed Navier-Stokes flows}

This section prepares a few lemmas for the proof of Theorem 1.3 in 6 . We first consider the solvability of the perturbed Navier-Stokes system (1.19) in Proposition 5.1 and Lemma 5.2, We then prove a few estimates in Lemmas 5.35 .4 .

We now recall a few notions related to self-similar solutions. The Navier-Stokes equations (1.1) in $I \times \Omega=(0, \infty) \times \mathbb{R}^{3}$ with zero force enjoy the scaling property that if $u(t, x)$ is a solution, then so are $u_{\lambda}(t, x):=\lambda u\left(\lambda^{2} t, \lambda x\right)$ for any $\lambda>0$. If $u_{\lambda}=u$ for all $\lambda>1$, then the solution is called forward self-similar (SS), and $u(t, x)=t^{-1 / 2} u\left(1, t^{-1 / 2} x\right)$. If $u_{\lambda}=u$ for a particular $\lambda>1$, then the solution is called forward discretely self-similar (DSS), and it is completely decided by its values when $T \leq t<T \lambda^{2}$ for any $T>0$. The existence of both type of solutions for small initial data $u_{0}$ with $u_{0}(x)=\lambda u_{0}(\lambda x)$ for all $\lambda>1$ or a particular $\lambda>1$, (also called SS or DSS), follows from Giga and Miyakawa [13, see also Cannone, Meyer and Planchon [5] and Cannone and Planchon [6].

We now state our result on the solvability of the perturbed Navier-Stokes system which is useful to describe the time-asymptotics of solutions for the initial-boundary value problem (1.1)-(1.3) in the next section. In particular, it implies the existence of the self-similar solution in Theorem 1.3 .

Proposition 5.1 (Perturbed system) For any $0<\eta<1$ there is $\varepsilon_{0}=\varepsilon_{0}(\eta)>0$ such that the following holds. Let $U, \tilde{U}, w_{0}$ be vector fields in $\mathbb{R}^{3}$ satisfying $\sup _{x}|x|\left(|U(x)|+|\tilde{U}(x)|+\left|w_{0}(x)\right|\right) \leq$ $\varepsilon \leq \varepsilon_{0}$ and $\operatorname{div} w_{0}=0$. Then there is a unique solution $w(t, x)$ of the perturbed Navier-Stoke system

$$
\partial_{t} w-\Delta w+\nabla(w \otimes w+U \otimes w+w \otimes \tilde{U})+\nabla p=0, \quad \operatorname{div} w=0
$$

in $\mathbb{R}^{3}$ with initial data $w(0)=w_{0}$, satisfying

$$
|w(t, x)| \lesssim \varepsilon(|x|+\sqrt{t})^{-1+\eta}|x|^{-\eta}
$$

The pressure satisfies $\|p(t)\|_{L^{s, \infty}\left(\mathbb{R}^{3}\right)} \lesssim \varepsilon^{2} t^{-1+\frac{3}{2 s}}$ for any $\frac{3}{2} \leq s \leq \frac{3}{1+\eta}$.

If furthermore $\sup _{x}|x|^{2}\left(|\nabla U(x)|+|\nabla \tilde{U}(x)|+\left|\nabla w_{0}(x)\right|\right) \leq \varepsilon$, then

$$
|\nabla w(t, x)| \lesssim \varepsilon(|x|+\sqrt{t})^{-1+\eta}|x|^{-1-\eta} .
$$

Comments for Proposition 5.1: 
1. $U$ and $\tilde{U}$ need not be divergence-free or self-similar. If we assume in the following that both $U$ and $\tilde{U}$ are SS, the solution set of (5.1) has the same scaling symmetry as the usual NavierStokes flows: If $w(t, x)$ is a solution of (5.1), then so is

$$
w_{\lambda}(t, x)=\lambda w\left(\lambda t, \lambda^{2} x\right)
$$

for any $\lambda>0$. By this scaling symmetry and the uniqueness part of Proposition [5.1, $w$ is forward SS (or forward DSS) if $w_{0}$ is SS (or DSS).

2. If $w$ is forward self-similar, then $w(t, x)=W(x / \sqrt{t}) / \sqrt{t}$ with $W(x)=w(1, x)$. In the case $U=\tilde{U}=0$ and $w_{0}$ is SS and small, we have $W \in L^{3, \infty}$ and expect $|W(y)| \leq \varepsilon\langle y\rangle^{-1}$, i.e.,

$$
|w(t, x)| \leq \varepsilon(|x|+\sqrt{t})^{-1} .
$$

3. For general initial data $\left|w_{0}(x)\right|<\varepsilon|x|^{-1}$ and $U=\tilde{U}=0$, we can construct solution of (5.1) satisfying the same bound (5.5). However, this is impossible if $U$ or $\tilde{U}$ is nonzero. See Remarks (i) and (ii) after the proof of Proposition 5.1

4. In the case $U$ and $\tilde{U}$ are nonzero, and all $U, \tilde{U}$ and $w_{0}$ are SS and small, $W(y)$ satisfies $\operatorname{div} W=0$ and

$$
-\frac{1}{2} W-\frac{1}{2} x \cdot \nabla W-\Delta W+\nabla(W \otimes W+U \otimes W+W \otimes \tilde{U})+\nabla P=0 .
$$

Since $W \in L^{3, \infty}$, we expect $|W(y)| \lesssim \varepsilon|y|^{-1}$ for large $y$. Due to the local singularity of $U$ and $\tilde{U}$, and the local analysis of [25], we can show $|W(y)| \lesssim \varepsilon|y|^{-\eta}$ for small $y$ and some $\eta>0$, with a smaller $\varepsilon$ needed if we want a smaller $\eta$. Thus

$$
|W(y)| \leq \varepsilon\left(|y|^{\eta}+|y|\right)^{-1},
$$

which corresponds to (5.2) for $w$. For general data $w_{0} \in X_{1}$, Proposition 5.1 asserts the unique existence of solutions $w$ satisfying the bound (5.2).

In order to prove Proposition 5.1 we start with a lemma.

Lemma 5.2 Let $n \in \mathbb{N}, b \geq 0, c \geq 0, b+c<n, \mu>0, \lambda \geq 0$, and $t>0$. Then

$$
\int_{\mathbb{R}^{n}}(|x-y|+\lambda)^{-b}|x-y|^{-c}(|y|+\sqrt{t})^{-n-\mu} d y \sim \sqrt{t}^{-\mu}(|x|+\lambda+\sqrt{t})^{-b}(x+\sqrt{t})^{-c} .
$$

Proof. We may assume $t=1$ since the general case follows from the change of variables $y=\sqrt{t} \hat{y}, x=\sqrt{t} \hat{x}$ and $\lambda=\sqrt{t} \hat{\lambda}$. Denote the integral with $t=1$ as $J(x)$ and $\rho=|x|+\lambda$. We want to show $J(x) \sim\langle\rho\rangle^{-b}\langle x\rangle^{-c}$. Let $a=\mu+b+c$. If $\rho<10$, we have

$$
J(x) \lesssim \int_{|y|<20}|x-y|^{-b-c} d y+\int_{|y|>20}|y|^{-n-a} d y \lesssim 1 .
$$

We also have $J(x) \gtrsim \int_{|y|>20}|y|^{-n-a} d y \gtrsim 1$. Assume now $\rho \geq 10$. We have

$$
J(x)=\int_{|y|<\rho / 4}+\int_{|y|>\rho / 4}=: J_{1}+J_{2} .
$$

For $J_{2}$, by replacing the factor $(|y|+1)$ of the integrand by $|y|$ and rescaling $y \rightarrow y / \rho$,

$$
J_{2} \leq \rho^{-a} \int_{|y| \geq 1 / 4}(|\hat{x}-y|+\hat{\lambda})^{-b}|\hat{x}-y|^{-c}|y|^{-n-a+b+c} d y,
$$

where $\hat{x}=x / \rho, \hat{\lambda}=\lambda / \rho$ and $|\hat{x}|+\hat{\lambda}=1$. The integral is of order 1 by the previous case $\rho<10$. Thus $J_{2} \lesssim \rho^{-a}$.

For $J_{1}$, because $|x-y|+\lambda \geq \rho / 4$ when $|y| \leq \rho / 4$,

$$
J_{1} \lesssim \rho^{-b} \int_{\mathbb{R}^{n}}|x-y|^{-c}\langle y\rangle^{-n-(a-b)+c} d y .
$$


Repeating the previous argument with $\lambda=0$ and $(b, a)$ replaced by $(0, a-b)$, we get $J_{1} \lesssim \rho^{-b}\langle x\rangle^{-c}$. We also have $J_{1} \gtrsim \rho^{-b} \int_{|y|<5 / 2}|x-y|^{-c} d y \gtrsim \rho^{-b}\langle x\rangle^{-c}$.

Since $\rho^{-a} \lesssim \rho^{-b}\langle x\rangle^{-c}$, we get $J(x) \sim \rho^{-b}\langle x\rangle^{-c}$.

\section{Proof of Proposition 5.1;} 2.55

Equation (5.1) can be written in the integral form using the heat kernel $\Gamma$ and the Stokes tensor

where

$$
w=w_{L}+w_{N}(w)
$$

and $F=F(w)=w \otimes w+U \otimes w+w \otimes \tilde{U}$. Since $e^{-p^{2}} \leq C_{k}\langle p\rangle^{-k}$ for any $k>0$, we can take $k=4$ and have

$$
\Gamma(t, y) \lesssim t^{-3 / 2}\langle x / \sqrt{t}\rangle^{-4}=\sqrt{t}(|x|+\sqrt{t})^{-4} .
$$

Suppose $\left|w_{0}(x)\right| \leq \varepsilon(|x|+\lambda)^{-1}, \lambda=0,1$. We have

$$
\left|w_{L}(t, x)\right| \lesssim \int \sqrt{t}(|y|+\sqrt{t})^{-4} \varepsilon(|x-y|+\lambda)^{-1} d y .
$$

By Lemma 5.2 with $(n, \mu, b, c)=(3,1,1,0)$,

$$
\left|w_{L}(t, x)\right| \lesssim \varepsilon(|x|+\lambda+\sqrt{t})^{-1} .
$$

Suppose further $\left|\nabla w_{0}(x)\right| \leq \varepsilon(|x|+\lambda)^{-2}$. By Lemma 5.2 again with $(n, \mu, b, c)=(3,1,2,0)$,

$$
\left|\nabla w_{L}(t, x)\right| \lesssim \int \sqrt{t}(|y|+\sqrt{t})^{-4} \varepsilon(|x-y|+\lambda)^{-2} d y \lesssim \varepsilon(|x|+\lambda+\sqrt{t})^{-2} .
$$

For $0<\eta<1$, define two norms for functions on $\mathbb{R}_{+} \times \mathbb{R}^{3}$ :

$$
\begin{gathered}
\|f\|_{\mathcal{Y}_{1}}=\sup _{(t, x) \in \mathbb{R}_{+} \times \mathbb{R}^{3}}\left[(|x|+\sqrt{t})^{1-\eta}|x|^{\eta}|f(t, x)|\right], \\
\|f\|_{\mathcal{Y}_{2}}=\|f\|_{\mathcal{Y}_{1}}+\sup _{(t, x) \in \mathbb{R}_{+} \times \mathbb{R}^{3}}\left[(|x|+\sqrt{t})^{1-\eta}|x|^{1+\eta}|\nabla f(t, x)|\right] .
\end{gathered}
$$

Estimates (5.18) and (5.19) show $\left\|w_{L}\right\|_{\mathcal{Y}_{1}} \leq C_{1} \varepsilon$ (resp. $\left\|w_{L}\right\|_{\mathcal{Y}_{2}} \leq C_{1} \varepsilon$ ) if $\left\|w_{0}\right\|_{X_{1}} \leq \varepsilon$ (resp. $\left\|w_{0}\right\|_{X_{1}}+$ $\left.\left\|\nabla w_{0}\right\|_{X_{2}} \leq \varepsilon\right)$ for some $C_{1}$.

We now estimate the nonlinear term $w_{N}(w)$. We will show

$$
\left\|w_{N}(w)-w_{N}(\tilde{w})\right\|_{\mathcal{Y}_{1}} \lesssim \varepsilon\|w-\tilde{w}\|_{\mathcal{Y}_{1}}
$$

if $\|w\|_{\mathcal{Y}_{1}} \leq \varepsilon$ and $\|\tilde{w}\|_{\mathcal{Y}_{1}} \leq \varepsilon$, and

$$
\left\|w_{N}(w)\right\|_{\mathcal{Y}_{2}} \lesssim \varepsilon\|w\|_{\mathcal{Y}_{2}}
$$

if $\|w\|_{\mathcal{Y}_{2}} \leq \varepsilon$. Note (5.22) implies $\left\|w_{N}(w)\right\|_{\mathcal{Y}_{1}} \lesssim \varepsilon\|w\|_{\mathcal{Y}_{1}}$ by taking $\tilde{w}=0$. These two estimates imply that the map

$$
w \rightarrow w_{L}+w_{N}(w)
$$

is a contraction mapping in the class of vector fields defined on $\mathbb{R}_{+} \times \mathbb{R}^{3}$ with $\|w\|_{\mathcal{Y}_{1}} \leq 2 C_{1} \varepsilon$ (resp. $\|w\|_{\mathcal{Y}_{2}} \leq 2 C_{1} \varepsilon$ ) if $\left\|w_{0}\right\|_{X_{1}} \leq \varepsilon$ (resp. $\left\|w_{0}\right\|_{X_{1}}+\left\|\nabla w_{0}\right\|_{X_{2}} \leq \varepsilon$ ) and $\varepsilon$ is sufficiently small.

If $\|w\|_{\mathcal{Y}_{1}} \leq \varepsilon,\|\tilde{w}\|_{\mathcal{Y}_{1}} \leq \varepsilon$, and $\|U\|_{X_{1}}+\|\tilde{U}\|_{X_{1}} \leq \varepsilon$, then

$$
|F(w)-F(\tilde{w})|(t, x) \lesssim \varepsilon\|w-\tilde{w}\|_{\mathcal{Y}_{1}}(|x|+\sqrt{t})^{-1+\eta}|x|^{-1-\eta} .
$$

If $\|w\|_{\mathcal{Y}_{2}} \leq \varepsilon$ and $\|U\|_{X_{1}}+\|\tilde{U}\|_{X_{1}}+\|\nabla U\|_{X_{2}}+\|\nabla \tilde{U}\|_{X_{2}} \leq \varepsilon$, then

$$
|\nabla F(w)|(t, x) \lesssim \varepsilon^{2}(|x|+\sqrt{t})^{-1+\eta}|x|^{-2-\eta} .
$$


Thus, using the definition of $w_{N}$ and $\nabla S$-estimate (2.56), to prove (5.22) and (5.23) it suffices to show

$$
\int_{0}^{t} \int_{\mathbb{R}^{3}}(|y|+\sqrt{s})^{-4}(|x-y|+\sqrt{t-s})^{-1+\eta}|x-y|^{-k-\eta} d y d s \lesssim(|x|+\sqrt{t})^{-1+\eta}|x|^{1-k-\eta}
$$

for $k=1,2$. Decompose the integral as

$$
I+I I:=\int_{0}^{\frac{t}{2}} \int_{\mathbb{R}^{3}} \cdots d y d s+\int_{\frac{t}{2}}^{t} \int_{\mathbb{R}^{3}} \cdots d y d s .
$$

We first estimate $I$. Since $(|x-y|+\sqrt{t-s}) \gtrsim t$

$$
I \lesssim \sqrt{t}^{-1+\eta} \int_{0}^{\frac{t}{2}} \int_{\mathbb{R}^{3}}(|y|+\sqrt{s})^{-4}|x-y|^{-k-\eta} d y d s .
$$

By Lemma 5.2 with $(n, \mu, b, c)=(3,1,0, k+\eta)$ and $t$ replaced by $s$,

$$
I \lesssim \sqrt{t}^{-1+\eta} \int_{0}^{\frac{t}{2}} \sqrt{s}^{-1}(|x|+\sqrt{s})^{-k-\eta} d s=\frac{t^{\frac{-1+\eta}{2}}}{|x|^{k+\eta-1}} \int_{0}^{\frac{t}{2 x^{2}}} \frac{d \tau}{\sqrt{\tau}(1+\sqrt{\tau})^{k+\eta}}
$$

where we used the scaling $s=|x|^{2} \tau$. Using $\int_{0}^{T} \frac{d \tau}{\sqrt{\tau}(1+\sqrt{\tau})^{k+\eta}} \lesssim \frac{\sqrt{T}}{\sqrt{T}+1}$ for $k+\eta>1$, (thus we need $\eta>0$ for $k=1$ ), we get

$$
I \lesssim \frac{t^{\frac{\eta}{2}}}{|x|^{k+\eta-1}} \frac{1}{(|x|+\sqrt{t})}
$$

which is bounded by $(|x|+\sqrt{t})^{-1+\eta}|x|^{1-k-\eta}$.

Next we estimate $I I$. Bounding the factor $(|y|+\sqrt{s})^{-4}$ by $(|y|+\sqrt{t})^{-4}$ and then integrating in time,

$$
\left.I I \lesssim \int_{\mathbb{R}^{3}}(|y|+\sqrt{t})^{-4}\left[(|x-y|+\sqrt{t / 2})^{1+\eta}-|x-y|\right)^{1+\eta}\right]|x-y|^{-k-\eta} d y
$$

Using $(A+B)^{1+\eta}-A^{1+\eta} \sim(A+B)^{\eta} B \sim A^{\eta} B+B^{1+\eta}$ for $A, B, \eta \geq 0$,

$$
\left.I I \lesssim \int_{\mathbb{R}^{3}}(|y|+\sqrt{t})^{-4}[|x-y|)^{-k} \sqrt{t}+|x-y|^{-k-\eta} \sqrt{t}^{1+\eta}\right] d y .
$$

By Lemma 5.2 with $(n, a, b)=(3,1,0)$ and $c=k$ or $c=k+\eta$,

$$
I I \lesssim(|x|+\sqrt{t})^{-k}
$$

which is also bounded by $(|x|+\sqrt{t})^{-1+\eta}|x|^{1-k-\eta}$. Summing up, we have shown (5.27) and thus the existence of $w$ satisfying (5.2) and (5.3).

It remains to show the estimate of the pressure, which follows from its equation

$$
-\Delta p=\partial_{j} \partial_{i}\left(w_{i} w_{j}+U_{i} w_{j}+w_{i} U_{j}\right) \quad \text { in } \mathbb{R}^{3},
$$

the Calderon-Zygmund estimates, and $F(w)$-estimate in (5.25) with $\tilde{w}=0$.

Remarks. (i) If $U=\tilde{U}=0$ and $\left|w_{0}(x)\right| \leq \varepsilon(|x|+\lambda)^{-1}$ with $\lambda=0$ or $\lambda=1$, then one can construct solutions in the class

$$
|w(t, x)| \lesssim \varepsilon(|x|+\lambda+\sqrt{t})^{-1}
$$

Indeed, in this case, we have $|F(t, x)| \lesssim \varepsilon^{2}(|x|+\lambda+\sqrt{t})^{-2}$ and can bound $w_{N}(w)(t, x)$ by $C \varepsilon^{2}(|x|+$ $\lambda+\sqrt{t})^{-1}$ using

$$
\int_{0}^{t} \int(|y|+\sqrt{s})^{-4}(|x-y|+\lambda+\sqrt{t-s})^{-2} d y d s \lesssim \sqrt{t}(|x|+\lambda+\sqrt{t})^{-2} .
$$


(ii) If $U$ or $\tilde{U}$ is nonzero, we need to take $\eta>0$ in (5.2). If $\eta=0$, we have $|F(t, x)| \lesssim \varepsilon^{2}(|x|+$ $\sqrt{t})^{-1}|x|^{-1}$ and need

$$
I=\int_{0}^{t} \int_{\mathbb{R}^{3}}(|y|+\sqrt{s})^{-4}(|x-y|+\sqrt{t-s})^{-1}|x-y|^{-1} d y d s \lesssim(x+\sqrt{t})^{-1} .
$$

However, when $|x| \ll \sqrt{t}$,

$$
I \gtrsim \int_{0}^{t / 2} \int_{|y|<\sqrt{t}}(|y|+\sqrt{s})^{-4}(\sqrt{t})^{-1}|x-y|^{-1} d y d s
$$

Integrating in $d s$ first,

$$
I \gtrsim \int_{|y|<\sqrt{t}}|y|^{-2}(\sqrt{t})^{-1}|x-y|^{-1} d y .
$$

Restricted in the subregion $2|x|<|y|<\sqrt{t}$,

$$
I \gtrsim \int_{2|x|<|y|<\sqrt{t}}|y|^{-3}(\sqrt{t})^{-1} d y \sim(\sqrt{t})^{-1} \log \frac{\sqrt{t}}{2|x|} .
$$

It is larger than $(x+\sqrt{t})^{-1}$ by a factor $\log \frac{\sqrt{t}}{2|x|}$ when $|x| \ll \sqrt{t}$.

(iii) Since $\nabla^{2} F \notin L_{x, l o c}^{1}$ and $\nabla^{2} S \notin L_{x, t, l o c}^{1}$, there is no suitable integral formula for $\nabla^{2} w$, and the above method does not allow us to estimate $\nabla^{2} w$ pointwise.

(iv) If all $U, \tilde{U}$ and $w_{0}$ are self-similar, then $w(t, x)=W(x / \sqrt{t}) / \sqrt{t}$ with $W(y)$ satisfying the elliptic equation (5.6), and one can estimate higher derivatives of $W$. It is not clear if $w_{0}$ is DSS.

In the rest of this section, we give two lemmas to be used in the next section.

Lemma 5.3 Let $\mathcal{R}_{\text {cut }}$ and cut-off function $\zeta$ be as in section 0 . There is a linear map

$$
\Lambda:\left\{w \in L_{l o c}^{1}\left(\mathbb{R}^{3} ; \mathbb{R}^{3}\right), \operatorname{div} w=0\right\} \rightarrow\left\{\hat{w} \in L_{l o c}^{1}\left(\mathbb{R}^{3} ; \mathbb{R}^{3}\right), \operatorname{supp} \hat{w} \subset \mathcal{R}_{\text {cut }}\right\}
$$

such that, for any $1<q<\infty$ and $q \leq r \leq \infty, \hat{w}=\Lambda w$ satisfies $\operatorname{div} \hat{w}=-\nabla \zeta \cdot w$ and

$$
\|\hat{w}\|_{W^{1, q, r}\left(\mathcal{R}_{c u t}\right)}+\|\nabla P \hat{w}\|_{L^{q, r}(\Omega)}+\|P \hat{w}\|_{L^{3 / 2, \infty}(\Omega) \cap L^{q_{b}, \infty}(\Omega)} \leq C_{q}\|w\|_{L^{q, r}\left(\mathcal{R}_{c u t}\right)}
$$

where $P$ is the Helmholtz projection on $L^{q, r}\left(\Omega ; \mathbb{R}^{3}\right), q_{b}=q^{*}$ if $q<3$, and $q_{b}=100$ if $q \geq 3$.

Proof. The usual construction of the solution of the problem div $u=f$ (see [10. Thm. III.3.1]) gives such a linear map with $\|\hat{w}\|_{W^{1, q}\left(\mathcal{R}_{\text {cut }}\right)} \leq C_{q}\|w\|_{L^{q}\left(\mathcal{R}_{\text {cut }}\right)}$. The bounds in Lorentz spaces follow from interpolation. It remains to show $\|P \hat{w}\|_{W^{1, q, r}(\Omega)} \leq C_{q}\|w\|_{L^{q, r}\left(\mathcal{R}_{\text {cut }}\right)}$. Decompose $\hat{w}=P \hat{w}+\nabla \pi$ where the scalar function $\pi$ solves the following Neumann boundary value elliptic problem:

$$
\Delta \pi=-\nabla \zeta \cdot w \quad \text { in } \Omega, \quad \nabla \pi \cdot N=0 \quad \text { on } \partial \Omega .
$$

Due to $L^{p}$ theory for elliptic equations with Neumann boundary condition, (by partition of unity and boundary estimates in [1, also see [32]), we have

$$
\left\|\nabla^{2} \pi\right\|_{L^{q, r}(\Omega)} \lesssim\|\nabla \zeta \cdot w\|_{L^{q, r}(\Omega)} \lesssim\|w\|_{L^{q, r}\left(\mathcal{R}_{c u t}\right)} .
$$

Combining estimates,

$$
\|\nabla P \hat{w}\|_{L^{q, r}(\Omega)} \lesssim\|\hat{w}\|_{W^{1, q, r}(\Omega)}+\left\|\nabla^{2} \pi\right\|_{L^{q, r}(\Omega)} \lesssim\|w\|_{L^{q, r}\left(\mathcal{R}_{c u t}\right)} .
$$

To bound $\|P \hat{w}\|_{L^{3 / 2, \infty}(\Omega) \cap L^{q_{b}}, \infty(\Omega)}$ amounts to bounding $\nabla \pi$ is in the same space. This can be shown by cut-off: $u=\pi \zeta$ is in $W_{l o c}^{2, q, r}, C_{l o c}^{2}$ outside of $\mathcal{R}_{c u t}$, and solves $\Delta u=f$ for some $f \in L^{q, r}$ with compact support. Thus $|\nabla u(x)| \leq C|x|^{-2}$ for $|x|$ large using Newtonian potential. This completes the proof.

Note: Although $f=\Delta u$ is a divergence, we do not know if $\int f=0$ (which would imply extra decay for $u$ ) since $\Delta u$ is not integrable and we cannot use divergence theorem. 
Lemma 5.4 Let $w$ be the solution in Proposition 5.1 with $w_{0}$ and $\nabla w_{0}$ satisfying the stated estimates. Let $\hat{w}=\Lambda w$ be defined by Lemma 5.3. Then, for all $3 \leq q \leq q_{1}$,

$$
\|\hat{w}(t)\|_{W^{1, q, \infty}}+\left\|\int_{0}^{t} e^{-(t-s) A} P \partial_{s} \hat{w}(s) d s\right\|_{L^{q, \infty}} \leq C \varepsilon\langle t\rangle^{-\sigma}
$$

where $\sigma=\frac{1+\delta}{2}-\frac{3}{2 q}>0$.

Proof. Denote $\mu=\frac{1-\eta}{2}$. Note $\sigma \leq \mu$ if $q \leq q_{1}$. By Lemma 5.3 and Proposition 5.1 .

$$
\|\hat{w}(t)\|_{W^{1, q, \infty}} \lesssim\|w(t)\|_{L^{q, \infty}\left(\mathcal{R}_{c u t}\right)} \lesssim\|w(t)\|_{L^{\infty}\left(\mathcal{R}_{c u t}\right)} \lesssim \varepsilon\langle t\rangle^{-\mu} .
$$

For the integral, using integration by parts, we have

$$
\int_{0}^{t} e^{-(t-s) A} P \partial_{s} \hat{w}(s) d s=P \hat{w}(t)-e^{-t A} P \hat{w}(0)-\mathcal{K}(t)
$$

where

$$
\mathcal{K}(t)=\int_{0}^{t} A^{\frac{1}{2}} e^{-(t-s) A} A^{\frac{1}{2}} P \hat{w}(s) d s .
$$

Choose $p$ so that $\mu=\frac{3}{2}\left(\frac{1}{p^{*}}-\frac{1}{q}\right)$, with $\frac{1}{p^{*}}=\frac{1}{p}-\frac{1}{3}$. (We need $q>\frac{3}{2(1-\mu)}=\frac{3}{1+\eta}$.) By Lemma 5.3 and Proposition 5.1

$$
\begin{gathered}
\|P \hat{w}(t)\|_{L^{q, \infty}} \lesssim\|\nabla P \hat{w}(t)\|_{L^{\frac{3 q}{q+3}, \infty}} \leq \varepsilon\langle t\rangle^{-\mu} . \\
\left\|e^{-t A} P \hat{w}(0)\right\|_{L^{q, \infty}} \lesssim t^{-\sigma}\|P \hat{w}(0)\|_{L^{\frac{3}{1+\delta}}, \infty} \lesssim \varepsilon t^{-\sigma},
\end{gathered}
$$

while for $t<1,\left\|e^{-t A} P \hat{w}(0)\right\|_{L^{q, \infty}} \lesssim\|P \hat{w}(0)\|_{L^{q}} \lesssim \varepsilon$.

To estimate $\mathcal{K}(t)$, let $Z=\left\{\varphi \in L_{\sigma}^{q^{\prime}, 1}:\|\varphi\|_{L_{\sigma}^{q^{\prime}, 1}} \leq 1\right\}$, with $1 / q+1 / q^{\prime}=1$ and $r=\left(q^{\prime}\right)^{*}$ i.e. $1 / r=1 / q^{\prime}-1 / 3$. We have

$$
\begin{gathered}
t^{\mu}\|\mathcal{K}(t)\|_{L^{q, \infty}}=\sup _{\varphi \in Z}\left(t^{\mu} \mathcal{K}(t), \varphi\right)=\sup _{\varphi}\left(t^{\mu} \int_{0}^{t}\left(s^{-\mu} A^{\frac{1}{2}} e^{-(t-s) A} \varphi, s^{\mu} A^{\frac{1}{2}} P \hat{w}(s)\right) d s\right. \\
\lesssim \sup _{\varphi} t^{\mu} \int_{0}^{t} s^{-\mu}\left\|A^{\frac{1}{2}} e^{-(t-s) A} \varphi\right\|_{L^{r, 1}} d s \sup _{s<t} s^{\mu}\left\|A^{\frac{1}{2}} P \hat{w}(s)\right\|_{L^{r^{\prime}, \infty}} \\
\lesssim \varepsilon \sup _{\varphi} t^{\mu} \int_{0}^{t} s^{-\mu}\left\|A^{\frac{1}{2}} e^{-(t-s) A} \varphi\right\|_{L^{r, 1}} d s \lesssim \varepsilon\|\varphi\|_{L^{q^{\prime}, 1}}
\end{gathered}
$$

where we used (2.13). This completes the proof.

\section{Time asymptotics}

In this section we consider large time asymptotics of the solution close to the periodic solution. We obtain Theorem 1.3 as a consequence of a more general result about the asymptotics:

Theorem 6.1 (Time asymptotics) For any $T>0, \delta>0, \eta>0$ and $3 \leq q_{1}<\frac{3}{\delta+\eta}$, there is $\varepsilon_{2}>0$ such that the following holds. Let $u_{*}$ and $f$ be time-periodic data satisfying (1.9). Let $u_{0} \in X_{1}$ be initial data satisfying $\left\|u_{0}\right\|_{L^{3, \infty}} \leq \varepsilon_{2}$. Assume that there exists a vector field $\tilde{u}_{0} \in X_{1}$ such that

$$
\left\|\tilde{u}_{0}\right\|_{X_{1}} \leq \varepsilon_{2}, \quad \nabla \tilde{u}_{0} \in X_{2} \quad \text { and } \quad \tilde{\varepsilon}:=\left\|u_{0}-\tilde{u}_{0}\right\|_{L^{3, \infty} \cap L^{\frac{3}{1+\delta}}, \infty} \leq \varepsilon_{2},
$$

then the solution $u$ in Theorem 1.1] (i) can be decomposed as the follows:

$$
u=Q+w+r .
$$

Here $Q$ is the periodic solution for the data $u_{*}$ and $f$ in Theorem 1.1 (iii). The term $w$ is the unique solution of the perturbed Navier-Stokes system (5.1) in $\mathbb{R}^{3}$ with $U=\tilde{U}=U^{b}$ and initial data $w_{0}=\tilde{u}_{0}-U^{b}$ in Theorem 5.1, where $U^{b}$ is the Landau solution corresponding to $Q$ given in Theorem 1.2 with $\alpha=1+\delta$. The term $r$ satisfies the following decay estimate:

$$
\|r(t)\|_{L_{w}^{q}(\Omega)} \leq C \tilde{\varepsilon} t^{-\frac{3}{2}\left(\frac{1}{3}-\frac{1}{q}\right)-\frac{\delta}{2}} \quad \forall t>0, \quad \forall q \in\left[3, q_{1}\right] .
$$




\section{Comments for Theorem 6.1:}

1. Theorem 1.3 is a special case of this theorem when $\tilde{u}_{0}$ is self-similar (or (-1)-homogeneous). We are also able to show that $w$ is DSS when $\tilde{u}_{0}$ is DSS as we mentioned in the comments of Proposition 5.1 .

2. The restriction $q \geq 3$ is related to the fact on the lack of the coercive estimate for the Stokes operator in $L^{p, \infty}(\Omega)$ for large $p$ in the exterior domain. See Lemma 2.1. Note that it is not the case when $\Omega=\mathbb{R}^{3}$ or when $\Omega$ is bounded.

3. By taking $\delta+\eta$ small, we can choose $q_{1}<\infty$ arbitrarily large. But our method does not allow $q_{1}=\infty$.

4. Our method allows us to consider $u_{*}=\tilde{u}_{*}+\hat{u}_{*}$ where $\tilde{u}_{*}$ is time periodic and $\hat{u}_{*}$ decays in time. We assume $\hat{u}_{*}=0$ for simplicity.

Proof. Suppose $\partial \Omega$ belongs to the region $0<R_{0}<|x|<R_{1}$ and let $\mathcal{R}_{\text {cut }}$ and cut-off function $\zeta(x)$ be defined as in section 3 To prove Theorem 6.1] we will study $z=u-Q-\tilde{w}$ instead of $r=u-Q-w$ where $\tilde{w}$ is a cut-off of $w$ which vanishes near $\partial \Omega$ :

$$
v=u-Q=\tilde{w}+z, \quad \tilde{w}=\zeta w+\hat{w},
$$

where $\hat{w}$ with compact support in $\mathcal{R}_{c u t}$ and $\operatorname{div} \hat{w}=-w \cdot \nabla \zeta$ is given by Lemma 5.3 . Since the difference $w-\tilde{w}$ is localized near the boundary, it is easy to show that it decays in time. Compared to (3.49), this choice ensures $\left.z\right|_{\partial \Omega}=0$ but introduces $\partial_{t} \hat{w}$ as a source term in $\partial_{t} z$ equation.

Specifically, $v$ and $w$ satisfy

$$
\begin{gathered}
\partial_{t} v-\Delta v+\nabla p_{1}=-\nabla F_{v}, \quad \operatorname{div} v=0, \\
\partial_{t} w-\Delta w+\nabla p_{0}=-\nabla F_{w}, \quad \operatorname{div} w=0,
\end{gathered}
$$

with

$$
F_{v}=(v+Q) \otimes v+v \otimes Q, \quad F_{w}=\left(w+U^{b}\right) \otimes w+w \otimes U^{b},
$$

and

$$
v(0)=v_{0}:=u_{0}-Q(0),\left.\quad v\right|_{\partial \Omega}=0, \quad w(0)=w_{0}:=\tilde{u}_{0}-U^{b} .
$$

Thus, $\tilde{w}$ defined by satisfies the Stokes system for $(t, x) \in \mathbb{R}_{+} \times \mathbb{R}^{3}$

$$
\partial_{t} \widetilde{w}-\Delta \widetilde{w}+\nabla\left(\zeta p_{0}\right)=-\nabla\left(\zeta F_{w}+\nabla \hat{w}\right)+\tilde{f}+\partial_{t} \hat{w}, \quad \operatorname{div} \tilde{w}=0,
$$

with $\tilde{w}(0)=\tilde{w}_{0}:=\zeta w_{0}+\hat{w}_{0}$, where

$$
\tilde{f}=(\nabla \zeta) \cdot F_{w}-2(\nabla \zeta \cdot \nabla) w-(\Delta \zeta) w+p_{0} \nabla \zeta
$$

The vector field $z=v-\tilde{w}=u-Q-\tilde{w}$, defined by (6.2), satisfies, for $p_{3}=p_{1}-\zeta p_{0}$,

$$
\begin{gathered}
\partial_{t} z-\Delta z+\nabla p_{3}=\nabla F_{z}-\tilde{f}-\partial_{t} \hat{w}, \quad \operatorname{div} z=0, \quad(x \in \Omega) \\
z(0)=z_{0},\left.\quad z\right|_{\partial \Omega}=0
\end{gathered}
$$

where $F_{z}=\zeta F_{w}-F_{v}+\nabla \hat{w}$ and

$$
z_{0}:=P\left(v_{0}-\tilde{w}_{0}\right)=P\left[\left(u_{0}-\tilde{u}_{0}\right)+\left(U^{b}-Q(0)\right)+\left((1-\zeta) w_{0}-\hat{w}_{0}\right)\right],
$$

and, using (1.15) with $\alpha=1+\delta$,

$$
\left\|z_{0}\right\|_{L^{3, \infty} \cap L^{\frac{3}{1+\delta}}, \infty} \lesssim \varepsilon .
$$

Note we can add the Helmholtz projection $P$ in the definition of $z_{0}$ since $z(0)=z_{0}$ is understood in weak sense. Since

$$
\begin{aligned}
F_{v}-F_{w}= & z \otimes z+(\tilde{w}+Q) \otimes z+z \otimes(\tilde{w}+Q)+\left(Q-U^{b}\right) \otimes \tilde{w}+\tilde{w} \otimes\left(Q-U^{b}\right) \\
& -(w-\tilde{w}) \otimes\left(w+U^{b}\right)-\left(\tilde{w}+U^{b}\right) \otimes(w-\tilde{w})
\end{aligned}
$$


we can decompose $F_{z}$ as

$$
F_{z}=z \otimes z+F_{1}(z)+F_{2}+F_{3}+F_{4}
$$

where

$$
\begin{aligned}
& F_{1}(z)=z \otimes(\tilde{w}+Q)+(\tilde{w}+Q) \otimes z, \\
& F_{2}=\tilde{w} \otimes\left(Q-U^{b}\right)+\left(Q-U^{b}\right) \otimes \tilde{w}, \\
& F_{3}=(w-\tilde{w}) \otimes\left(w+U^{b}\right)-\left(\tilde{w}+U^{b}\right) \otimes(w-\tilde{w}), \\
& F_{4}=(\zeta-1) F_{w}+\nabla \hat{w} .
\end{aligned}
$$

Note that $F_{3}$ and $F_{4}$ have compact supports.

We will prove

$$
\sup _{t>0} t^{\sigma}\|z(t)\|_{L^{q, \infty}} \lesssim \varepsilon \quad \text { for } \quad q \in\left[3, q_{1}\right],
$$

with

$$
\sigma=\sigma(q, \delta)=\frac{3}{2}\left(\frac{1}{3}-\frac{1}{q}\right)+\frac{\delta}{2}>0 .
$$

By unique existence of $u \in L^{\infty} L^{3, \infty}$ (Theorem 1.1) and Hölder inequality, it suffices to prove the existence of $z$ satisfying (6.9), (6.10) and

$$
\|z\|_{Z}:=\sup _{t>0}\left\{t^{\delta / 2}\|z(t)\|_{L^{3, \infty}}+t^{\sigma_{1}}\|z(t)\|_{L^{q_{1}, \infty}}\right\} \lesssim \varepsilon
$$

where $\sigma_{1}=\sigma\left(q_{1}, \delta\right)$.

Denote

$$
\Psi(f)(t)=\int_{0}^{t} e^{-(t-s) A} \operatorname{Pf}(s) d s
$$

where the integral is in the weak sense,

$$
(\Psi(f)(t), \varphi)=\int_{0}^{t}\left(f(s), e^{-(t-s) A} \varphi\right) d s, \quad \forall \varphi \in C_{c, \sigma}^{\infty}(\Omega) .
$$

A solution $z(t)$ of $(6.9)$ and $(6.10)$ is a fixed point of the nonlinear map $\Phi$ defined for $z \in Z$,

$$
\Phi z(t)=z^{1}(t)+\Psi\left(\nabla\left[z \otimes z+F_{1}(z)\right]\right)(t)
$$

where

$$
z^{1}(t)=e^{-t A} z_{0}+\Psi\left(\nabla\left[F_{2}+F_{3}+F_{4}\right]-\tilde{f}-\partial_{t} \hat{w}\right)(t)
$$

We will show that for $\varepsilon$ sufficiently small,

$$
\|\Phi z\|_{Z} \leq C \varepsilon, \quad\|\Phi z-\Phi \tilde{z}\|_{Z} \leq C \varepsilon\|z-\tilde{z}\|_{Z},
$$

which guarantees the unique existence of a fixed point $z$ of $\Phi$ with $\|z\|_{Z} \leq C \varepsilon$.

By Lemma 2.2 (i), we have

$$
t^{\sigma}\left\|e^{-t A} z_{0}\right\|_{L^{q, \infty}} \lesssim\left\|z_{0}\right\|_{L^{\frac{3}{1+\delta}}, \infty}
$$

Consider the terms of divergence form. By duality, we have

$$
\|\Psi(\nabla F)\|_{L^{q, \infty}}=\sup _{\varphi \in L_{\sigma}^{q^{\prime}, 1}}|(\Psi(\nabla F), \varphi)| .
$$

By (2.13) with $1 / r=1 / q^{\prime}-1 / 3$ and $1 / r^{\prime}=1 / q+1 / 3$, we have

$$
\begin{aligned}
|(\Psi(\nabla F), \varphi)| & \leq \int_{0}^{t}\|F(s)\|_{L^{r^{\prime}, \infty}}\left\|\nabla e^{-(t-s) A} \varphi\right\|_{L^{r, 1}} d s \\
& \lesssim\left(\sup _{t>0} t^{\sigma}\|F(t)\|_{L^{r^{\prime}, \infty}}\right) \int_{0}^{t} s^{-\sigma}\left\|\nabla e^{-(t-s) A} \varphi\right\|_{L^{r, 1}} d s \\
& \lesssim\left(\sup _{t>0} t^{\sigma}\|F(t)\|_{L^{r^{\prime}, \infty}}\right) t^{-\sigma}\|\varphi\|_{L^{q^{\prime}, 1}} .
\end{aligned}
$$


In the above we need $1<q^{\prime} \leq 3 / 2$, thus $3 \leq q<\infty$.

We now estimate $F=F_{z}$. First,

$$
t^{\sigma}\left\|z \otimes z+F_{1}(z)\right\|_{L^{r^{\prime}, \infty}} \lesssim t^{\sigma}\|z\|_{q, \infty}\left(\|z\|_{3, \infty}+\|\tilde{w}\|_{3, \infty}+\|Q\|_{3, \infty}\right) \lesssim \varepsilon^{2} .
$$

Next, since $w-\tilde{w}=(1-\zeta) w+\hat{w}$ is compactly supported, it follows from Lemma 5.3 and Proposition 5.1 that, for $1<q<\infty$,

$$
\begin{aligned}
\|w-\tilde{w}\|_{L^{q, \infty}(\Omega)} & \lesssim\|w(1-\zeta)\|_{q, \infty}+\|\hat{w}\|_{q, \infty} \\
& \lesssim\|w(1-\zeta)\|_{q, \infty}+\|w\|_{L^{q, \infty}\left(\mathcal{R}_{c u t}\right)} \\
& \lesssim\|w\|_{L^{\infty}(\Omega)} \lesssim \varepsilon\langle t\rangle^{-\frac{1-\eta}{2}}
\end{aligned}
$$

Also note

$$
\|w(t)\|_{L^{r, \infty}\left(\mathbb{R}^{3}\right)} \lesssim \varepsilon t^{-\frac{1}{2}+\frac{3}{2 r}}, \quad(3 \leq r \leq 3 / \eta) .
$$

With $1 / q_{b}=1 / q-\delta / 3$, (hence $\frac{1}{2}-\frac{3}{2 q_{\mathrm{b}}}=\sigma(q, \delta)$ ), we have

$$
\begin{aligned}
\left\|F_{2}\right\|_{L^{r^{\prime}, \infty}} & \lesssim\left\|Q-U^{b}\right\|_{\frac{3}{1+\delta}, \infty}\|\tilde{w}\|_{q_{b}, \infty} \lesssim \varepsilon\left(\|w\|_{q_{b}, \infty}+\varepsilon\langle t\rangle^{-\frac{1-\eta}{2}}\right) \\
& \lesssim \varepsilon^{2}\left(t^{-\sigma}+\langle t\rangle^{-\frac{1-\eta}{2}}\right) \lesssim \varepsilon^{2} t^{-\sigma} .
\end{aligned}
$$

Recall we take $\alpha=1+\delta$ in Theorem 1.2 so that $Q-U^{b} \in L^{\frac{3}{1+\delta}, \infty}$. We have also used that $0<\sigma \leq \frac{1-\eta}{2}$ for $3 \leq q \leq q_{1}$, which also implies

$$
\|w-\tilde{w}\|_{L^{q, \infty}(\Omega)} \lesssim \varepsilon t^{-\sigma}
$$

Thus

$$
t^{\sigma}\left\|F_{3}\right\|_{r^{\prime}, \infty} \lesssim t^{\sigma}\|w-\tilde{w}\|_{q, \infty}\left(\|w\|_{3, \infty}+\|\tilde{w}\|_{3, \infty}+\left\|U^{b}\right\|_{3, \infty}\right) \lesssim \varepsilon^{2} .
$$

Finally $F_{4}=(\zeta-1) F_{w}+\nabla \hat{w}$ can be estimated as follows:

$$
\begin{aligned}
\left\|F_{4}\right\|_{r^{\prime}, \infty} & \lesssim\left(\|w\|_{3, \infty}+\left\|U^{b}\right\|_{3, \infty}\right)\|(\zeta-1) w\|_{q, \infty}+\|w\|_{L^{r^{\prime}, \infty}\left(\mathcal{R}_{c u t}\right)} \\
& \lesssim\|w\|_{L^{\infty}(\Omega)} \lesssim \varepsilon\langle t\rangle^{-\frac{1-\eta}{2}} \lesssim \varepsilon t^{-\sigma} .
\end{aligned}
$$

Next we consider $\Psi(\tilde{f})$. Denote $r_{1}=\frac{3}{2+\delta+\eta}$ and $r_{2}=\frac{3}{1+\eta}$. Note $1<r_{1}<r_{2}$. By decay estimates,

$$
\|\Psi(\tilde{f})(t)\|_{q, \infty} \lesssim \int_{0}^{t}(t-s)^{-\sigma_{2}}\|\tilde{f}(s)\|_{r_{1}, \infty} d s, \quad \sigma_{2}=\frac{3}{2}\left(\frac{1}{r_{1}}-\frac{1}{q}\right) .
$$

Note $0<\sigma_{2}<1$ since $q \leq q_{1}<\frac{3}{\delta+\eta}$. By Proposition 5.1, on $\mathcal{R}_{\text {cut }}$ we have $|w|+|\nabla w| \lesssim \varepsilon\langle t\rangle{ }^{-\frac{1}{2}+\frac{\eta}{2}}$, $\left|F_{w}\right| \lesssim|w|^{2}+\varepsilon|w| \lesssim \varepsilon^{2}\langle t\rangle^{-\frac{1}{2}+\frac{\eta}{2}}$ and $\left\|p_{0}(t)\right\|_{r_{2}, \infty} \leq \varepsilon^{2}\langle t\rangle^{-\frac{1}{2}+\frac{\eta}{2}}$. Thus, using supp $\tilde{f}(t) \subset \mathcal{R}_{c u t}$,

$$
\|\tilde{f}(s)\|_{r_{1}, \infty} \lesssim\|\tilde{f}(s)\|_{r_{2}, \infty} \lesssim \varepsilon\langle s\rangle^{-\frac{1}{2}+\frac{\eta}{2}}
$$

We get

$$
\|\Psi(\tilde{f})(t)\|_{q, \infty} \lesssim \int_{0}^{t}(t-s)^{-\sigma_{2}} \varepsilon\langle s\rangle^{-\frac{1}{2}+\frac{\eta}{2}} d s \lesssim \varepsilon t^{-\sigma_{2}+\frac{1}{2}+\frac{\eta}{2}}=\varepsilon t^{-\sigma} .
$$

Finally the estimate $\Psi\left(\partial_{t} \hat{w}\right)$ is proved by Lemma 5.4

The above shows the first assertion $\|\Phi z\|_{Z} \leq C \varepsilon$ in (6.23). The second estimate in (6.23) on $\|\Phi z-\Phi \tilde{z}\|_{Z}$ is proved similarly. This finishes the proof of Theorem 6.1.

\section{Acknowledgments}

We thank Professor C.-C. Chen for providing the references [1, 32, for (5.45), and Professor Y. Giga for discussing Lemma 2.2 (ii). Part of this work was done when all of us visited the National Center for Theoretical Sciences (Taipei Office) and the Department of Mathematics, National Taiwan University, when both Kang and Miura visited the University of British Columbia, and when Tsai 
visited Sungkyunkwan University and Taida Institute for Mathematical Sciences. We would like to thank the kind hospitality of these institutions and Professors D. Chae, C.-C. Chen, S. Gustafson, J. Lee, C.-S. Lin and C.-L. Wang. The research of Kang is partly supported by KRF-2008-331C00024 and R01-2008-000-11008-0. The research of Miura was partly supported by the JSPS grant no. 191437. The research of Tsai is partly supported by Natural Sciences and Engineering Research Council of Canada, grant no. 261356-08.

\section{References}

[1] Agmon, S.; Douglis, A.; Nirenberg, L.: Estimates near the boundary for solutions of elliptic partial differential equations satisfying general boundary conditions. Comm. Pure Appl. Math. $121959623-727$.

[2] Amann, H.: Navier-Stokes Equations with Nonhomogeneous Dirichlet Data, Journal of Nonlinear Mathematical Physics Volume 10, Supplement 1 (2003), 1-11.

[3] Borchers, W.; Miyakawa, T.: On stability of exterior stationary Navier-Stokes flows. Acta Math. 174 (1995), no. 2, 311-382.

[4] Cannone, M.; Karch, G.: Smooth or singular solutions to the Navier-Stokes system? J. Differential Equations 197 (2004), no. 2, 247-274.

[5] Cannone, M.; Meyer, Y.; Planchon, F.: Solutions auto-simlaires des equations de Navier-Stokes, In: Seminaire X-EDP, Centre de Mathematiques, Ecole polytechnique, 1993-1994.

[6] Cannone, M.; Planchon, F.: Self-similar solutions for the Navier-Stokes equations in $R^{3}$. Comm. Part. Diff. Equ. 21 (1996), no. 1-2, 179-193.

[7] Cazenave, T., Dickstein, F.,Weissler, F.: Chaotic behavior of solutions of the Navier-Stokes system in $R^{N}$. Adv. Differ. Equ. 10 (4), 361-398, 2005.

[8] Farwig, R.; Kozono, H.; Sohr, H.: Very weak solutions of the Navier-Stokes equations in exterior domains with nonhomogeneous data. J. Math. Soc. Japan 59 (2007), no. 1, 127-150.

[9] Finn, R.: On the exterior stationary problem for the Navier-Stokes equations, and associated perturbation problems. Arch. Rational Mech. Anal. 191965 363-406.

[10] Galdi, G. P.: An Introduction to the Mathematical Theory of the Navier-Stokes Equations: Linearized steady problems, Volume I, Springer, 1994.

[11] Galdi, G. P.: An Introduction to the Mathematical Theory of the Navier-Stokes Equations: Nonlinear steady problems, Volume II, Springer, 1994.

[12] Galdi, G.; Sohr, H.: Existence and uniqueness of time-periodic physically reasonable NavierStokes flow past a body. Arch. Ration. Mech. Anal. 172 (2004), no. 3, 363-406.

[13] Giga, Y.; Miyakawa, T.: Navier-Stokes Flow in $R^{3}$ with Measures as Initial Vorticity and Morrey Spaces. Comm. Part. Differ. Equ. 14 (5), 577-618, 1989.

[14] Heywood, J. G.: The exterior nonstationary problem for the Navier-Stokes equations. Acta Math. 129 (1972), no. 1-2, 11-34.

[15] Kapitanskii, L. V.; Piletskas, K. I.: Some problems of vector analysis. (Russian) Boundary value problems of mathematical physics and related problems in the theory of functions, 16. Zap. Nauchn. Sem. Leningrad. Otdel. Mat. Inst. Steklov. (LOMI) 138 (1984), 65-85.

[16] Kim, H.; Kozono, H.: A removable isolated singularity theorem for the stationary Navier-Stokes equations. Journal of Differential Equations 220 (2006), 68-84.

[17] Korolev, A.; Sverak, V.: On the large-distance asymptotics of steady state solutions of the Navier-Stokes equations in 3D exterior domains, preprint: arXiv:0711.0560 
[18] Kozono, H.; Nakao, M.: Periodic solutions of the Navier-Stokes equations in unbounded domains. Tohoku Math. J. (2) 48, 33-50 (1996).

[19] Kozono, H.; Ogawa, T.: On stability of Navier-Stokes flows in exterior domains. Arch. Rational Mech. Anal. 128 (1994), no. 1, 1-31.

[20] Kozono, H.; Yamazaki, M.: On a larger class of stable solutions to the Navier-Stokes equations in exterior domains. Math. Z. 228 (1998), no. 4, 751-785.

[21] Kozono, H.; Yanagisawa, T.: Nonhomogeneous boundary value problems for stationary NavierStokes equations in a multiply connected bounded domain. Pacific J. Math. 243 (2009), no. 1, 127-150.

[22] Landau, L. D.: A new exact solution of the Navier-Stokes equations, Dokl. Akad. Nauk SSSR, 43, 299, 1944.

[23] Landau, L. D.; Lifshitz, E. M.: Fluid Mechanics, second edition, Butterworth-Heinemann, 2000 paperback reprinting.

[24] Maremonti, P.; Padula, M.: Existence, uniqueness and attainability of periodic solutions of the Navier-Stokes equations in exterior domains. (English, Russian summary) Zap. Nauchn. Sem. S.-Peterburg. Otdel. Mat. Inst. Steklov. (POMI) 233 (1996), Kraev. Zadachi Mat. Fiz. i Smezh. Vopr. Teor. Funkts. 27, 142-182, 257; translation in J. Math. Sci. (New York) 93 (1999), no. 5, 719-746.

[25] Miura, H.; Tsai, T.-P.: Point singularities of 3D stationary Navier-Stokes flows, J. Math. Fluid Mech., DOI: 10.1007/s00021-010-0046-6

[26] Miyakawa, T.: On nonstationary solutions of the Navier-Stokes equations in an exterior domain. Hiroshima Math. J. 12 (1982), no. 1, 115-140.

[27] Nazarov, S. A., Pileckas, K.: On steady Stokes and Navier-Stokes problems with zero velocity at infinity in a three-dimensional exterior domain, J. Math. Kyoto Univ. 40-3 (2000), 475-492.

[28] Oseen, C. W.: Hydrodynamik, Leipzig, 1927.

[29] Planchon, F.: Asymptotic behavior of global solutions to the Navier-Stokes equations in $R^{3}$. Rev. Mat. Iberoamericana 14 (1998), no. 1, 71-93.

[30] Salvi, R.: On the existence of periodic weak solutions on the Navier-Stokes equations in exterior regions with periodically moving boundaries. Navier-Stokes equations and related nonlinear problems (Funchal, 1994), 63-73, Plenum, New York, 1995.

[31] Simader, C. G.; Sohr, H.: A new approach to the Helmholtz decomposition and the Neumann problem in Lq spaces for bounded and exterior domains. In: G.P. Galdi, Editor, Math. Probl. Relating to the Navier-Stokes Equations, World Scientific, Singapore (1992).

[32] Smith, H. F.: The subelliptic oblique derivative problem. Comm. Partial Differential Equations 15 (1990), no. $1,97-137$.

[33] Solonnikov, V. A.: Estimates for solutions of a non-stationary linearized system of Navier-Stokes equations. (Russian) Trudy Mat. Inst. Steklov. 70 (1964) 213-317.

[34] Sverak, V.: On Landau's Solutions of the Navier-Stokes Equations, preprint: arXiv:math/0604550

[35] Taniuchi, Y.: On the uniqueness of time-periodic solutions to the Navier-Stokes equations in unbounded domains. Math. Z. 261 (2009), no. 3, 597-615.

[36] Tian, G.; Xin, Z.: One-point singular solutions to the Navier-Stokes equations. Topol. Methods Nonlinear Anal. 11 (1998), no. 1, 135-145.

[37] Yamazaki, M.: The Navier-Stokes equations in the weak- $L^{n}$ space with time-dependent external force. Math. Ann. 317 (2000), no. 4, 635-675. 